\title{
Effect of the counterrotating-wave terms on the spontaneous emission from a multilevel atom
}

\author{
Zheng-Hong Li, ${ }^{1}$ Da-Wei Wang, ${ }^{2}$ Hang Zheng, ${ }^{3}$ Shi-Yao Zhu, ${ }^{1,2}$ and M. Suhail Zubairy ${ }^{4}$ \\ ${ }^{1}$ Department of Physics, Hong Kong Baptist University, Hong Kong, China \\ ${ }^{2}$ Department of Physics and ITP, The Chinese University of Hong Kong, Hong Kong, China \\ ${ }^{3}$ Department of Physics, Shanghai Jiao Tong University, Shanghai 200030, China \\ ${ }^{4}$ Department of Physics and Institute for Quantum Studies, Texas A\&M University, College Station, Texas 77843, USA
}

(Received 28 April 2009; published 3 August 2009)

\begin{abstract}
The spontaneous decay of a multilevel atom interacting with the electromagnetic field in free space is investigated with a unitary transformation method, which is introduced in order to include all rotating and counter-rotating terms in the Hamiltonian. By using the ground state of the total Hamiltonian, the evolution of the effective decay rate and the energy shift are calculated. When the atomic transition frequency is smaller than the central frequency of the spectrum, the Zeno effect dominates, and if the atomic transition frequency is larger than the central frequency, the anti-Zeno effect will dominate. The time evolution of the energy shift is obtained. The counter-rotating terms lead to a shift toward the low frequency region for the frequency distribution of the emitted photon.
\end{abstract}

DOI: 10.1103/PhysRevA.80.023801

PACS number(s): $42.50 . \mathrm{Ct}$

\section{INTRODUCTION}

It is well known that the counter-rotating terms are not important in the resonant interaction between an atomic system and an electromagnetic field and also in the spontaneous emission in the long-time limit. However, the counterrotating terms are important for the energy shift [1-3] and it was recently found that, for the short time, the dynamic evolutions of the spontaneous emission without and with the rotating wave approximation (RWA) are different $[1,4]$. In this time regime, the quantum Zeno and quantum anti-Zeno effects [5-7] for the spontaneous emission from an atom are deeply affected by the counter-rotating terms. For the hydrogen atom, due to the counter-rotating terms, the Zeno time is longer by two orders of magnitude than that obtained with RWA and there is no anti-Zeno effect [1]. Consequently, the experimental measurement of the quantum Zeno effect may be much easier than what was determined with the RWA [8-11]. Recently the quantum Zeno and anti-Zeno effects in spontaneous decay have been used in the investigation of how to control the thermodynamic evolution [12].

In this paper, we present detailed calculations and present the analytical study for the multilevel atom coupled to the electromagnetic field in free space without making the RWA. Our approach in which the Hamiltonian that contains the counter-rotating terms is reduced to a Hamiltonian of the same form as obtained with RWA allows us to handle many problems of interest. An interesting question related to the ground state of the whole system, as the atom is always immersed in the vacuum reservoir, is addressed in a more natural and simple way. The effect of the counter-rotating terms for the system coupled to a thermal bath was discussed in $[4,7,12]$, but they are all based on an improper ground state, the ground state of the bare atom with no photon. The energy of this ground state of the bare atom is higher than the ground state of the whole system [13]; see Appendix A. If we put the atom in the ground state of the bare atom, we find that it evolves into a state with population in the excited state of the bare atom due to the interaction with the vacuum (with entropy increasing); see Appendix A. We also find that the energy of the excited state of the bare atom is higher than the energy of the excited state of the whole system (Appendix A). Therefore, instead of starting with the ground state of the bare atom, we start with the ground state of the whole system, which can be obtained with our unitary transformation method. For our studies, on the spontaneous emission, we consider the atom to be initially in an excited state which is obtained by acting a Hermitian operator on the ground state of the whole system. The time evolution of the system including the population decay and the energy shifts of the excited and the ground states are obtained. The dependence of the quantum Zeno and anti-Zeno effects on the transition frequency and the spectrum of the vacuum are investigated. The contribution to the energy shift from the emission and reabsorption of virtual photons with the vacuum and from the emission of a real photon is discussed and the time dependence of the energy shift is calculated. The spectrum of the field emitted by the atom is discussed which is different from that obtained in previous methods. The method used in this paper is convenient to calculate a lot of problems where RWA is not valid such as the nonresonant light scattering problems besides the short-time evolution.

\section{BASIC THEORY}

The interaction between a multilevel atom and the vacuum reservoir can be described by the Hamiltonian (setting $\hbar=1$ )

$$
\begin{gathered}
H=H_{0}+H_{1}, \\
H_{0}=\sum_{i} \omega_{i}|i\rangle\langle i|+\sum_{\mathbf{k}} \omega_{k} b_{\mathbf{k}}^{\dagger} b_{\mathbf{k}}, \\
H_{1}=\sum_{i, j \neq i, \mathbf{k}} g_{\mathbf{k}, i j}\left(b_{\mathbf{k}}^{\dagger}+b_{\mathbf{k}}\right)|i\rangle\langle j|,
\end{gathered}
$$

where $\omega_{i}$ is the energy of the $|i\rangle$ state, $b_{\mathbf{k}}^{\dagger}\left(b_{\mathbf{k}}\right)$ is the creation (annihilation) operator of the kth EM mode with frequency 
$\omega_{k}(k=|\mathbf{k}|)$, and $\hat{\mathbf{e}}_{\mathbf{k}}$ is the unit polarization vector. Here $g_{\mathbf{k}, i j}$ $=-\frac{e}{m} \sqrt{\frac{1}{2 \varepsilon_{0} \omega_{k} L^{3}}} \hat{\mathbf{e}}_{\mathbf{k}} \cdot \mathbf{p}_{i j}$ is the coupling constant between the atomic transition $(|i\rangle \leftrightarrow|j\rangle)$ and the kth EM mode with $\mathbf{p}_{i j}$ being the transition matrix element of the momentum operator. The interacting spectrum is defined by $[8,9]$

$$
G_{i j}(\omega)=\sum_{\mathbf{k}} g_{\mathbf{k}, i j}^{2} \delta\left(\omega-\omega_{k}\right)
$$

Please note that the ground state (the eigenstates) of $H_{0}$ is not the ground state (eigenstates) of the total Hamiltonian due to the counter-rotating terms. When an atom is put in the free vacuum, the atom is in the ground state of the total system [13]. Starting with the ground state (or an eigenstate) of $H_{0}$ is not realistic.

The above Hamiltonian includes both the rotating terms and counter-rotating terms. In order to eliminate the counterrotating terms in the interaction Hamiltonian $H_{1}$, we make the following unitary transformation of, $H^{S}=\exp (i S) H \exp ($ $-i S)$, with

$$
S=\sum_{i, j \neq i, \mathbf{k}} \frac{g_{\mathbf{k}, i j} \xi_{k, i j}}{i \omega_{k}}\left(b_{\mathbf{k}}^{\dagger}-b_{\mathbf{k}}\right)|i\rangle\langle j|
$$

Here $\xi_{k, i j}$, which depends on $k$ and $\omega_{i j}=\omega_{i}-\omega_{j}$, is chosen such that the counter-rotating terms cancel out $[1,14]$. For this purpose, we expand $H^{S}$ in a power series of $g_{\mathbf{k}, i j}$, i.e., $H^{S}=H_{0}+H_{1}^{S}+H_{2}^{S}+O\left(g_{k}^{3}\right)$, where $O\left(g_{\mathbf{k}}^{3}\right)$ contains terms of the third and higher orders in $g_{\mathbf{k}}$ and will be neglected.

The first-order term in $g_{\mathbf{k}, i j}$ is

$$
H_{1}^{S}=H_{1}+\left[i S, H_{0}\right] \text {. }
$$

It follows from the definition of $S$ and the Hamiltonians $H_{0}$ and $H_{1}$ that

$$
\begin{aligned}
H_{1}^{S}= & \sum_{i, j \neq i, \mathbf{k}} g_{\mathbf{k}, i j} \xi_{k, i j}\left\{\left[\omega_{j i}\left(b_{\mathbf{k}}^{\dagger}-b_{\mathbf{k}}\right) \frac{1}{\omega_{k}}-\left(b_{\mathbf{k}}^{\dagger}+b_{\mathbf{k}}\right)\right]\right\}|i\rangle\langle j| \\
& +\sum_{i, j \neq i, \mathbf{k}} g_{\mathbf{k}, i j}\left(b_{\mathbf{k}}^{\dagger}+b_{\mathbf{k}}\right)|i\rangle\langle j| \\
= & \sum_{i, j, \mathbf{k}} g_{\mathbf{k}, i j}\left(\frac{\xi_{k, i j}}{\omega_{k}} \omega_{j i}-\xi_{k, i j}+1\right)\left(b_{\mathbf{k}}^{\dagger}|i\rangle\left\langle j\left|+b_{\mathbf{k}}\right| j\right\rangle\langle i|\right) \\
& +\sum_{i, j, \mathbf{k}}^{i<j} g_{\mathbf{k}, i j}\left(\frac{\xi_{k, i j}}{\omega_{k}} \omega_{i j}-\xi_{k, i j}+1\right)\left(b_{\mathbf{k}}^{\dagger}|j\rangle\left\langle i\left|+b_{\mathbf{k}}\right| i\right\rangle\langle j|\right),
\end{aligned}
$$

where the second term is the counter-rotating one. For the purpose of eliminating the counter-rotating terms in $H_{1}^{S}$, we choose $\frac{\xi_{k, i j}}{\omega_{k}} \omega_{i j}-\xi_{k, i j}+1=0$ for $\omega_{i j}<0$. This leads to the choice

$$
\xi_{k, i j}=\frac{\omega_{k}}{\left|\omega_{i j}\right|+\omega_{k}}
$$

The coefficient of the first term becomes (note $\omega_{i j}=-\omega_{j i}$ )

$$
\frac{\xi_{k, i j}}{\omega_{k}} \omega_{j i}-\xi_{k, i j}+1=\frac{2 \xi_{k, i j}}{\omega_{k}} \omega_{j i}
$$

and consequently, $H_{1}^{S}$ has the same form as the interaction Hamiltonian of the RWA

$$
H_{1}^{S}=\sum_{i, j, \mathbf{k}}^{i<j} \frac{2 g_{\mathbf{k}, i j} \xi_{k, i j}}{\omega_{k}} \omega_{j i}\left(b_{\mathbf{k}}^{\dagger}|i\rangle\left\langle j\left|+b_{\mathbf{k}}\right| j\right\rangle\langle i|\right)
$$

with the new coupling constant $\frac{2 g_{\mathbf{k}, i j} \xi_{k, i j}}{\omega_{k}} \omega_{j i}$.

The second order $H_{2}^{S}=\left[i S, H_{1}\right]+\frac{1}{2}\left[i S,\left[i S, H_{0}\right]\right]$ can be written as

$$
H_{2}^{S}=-\sum_{i, j, \mathbf{k}}^{i \neq j} \frac{g_{\mathbf{k}, i j}^{2}}{\omega_{k}}\left\{2 \xi_{k, i j}-\xi_{k, i j}^{2}-\frac{\xi_{k, i j}^{2}}{\omega_{k}}\left[\omega_{j}-\omega_{i}\right]\right\}|i\rangle\langle i|,
$$

where we have dropped all the nondiagonal terms $|i\rangle\langle j|(i$ $\neq j$ ), whose contribution to physical quantities is of the fourth order in $g_{\mathbf{k}}$. The details of the calculation can be found in Appendix B.

The self-energy of a free electron $[2,14]$ is due to the exchange of virtual photons with the vacuum and is given by $E_{s e}=-\frac{1}{4 \pi \varepsilon_{0}} \frac{4 e^{2}}{3 \pi m c^{3}} \int_{0}^{\omega_{c}} \frac{\mathbf{p}^{2}}{2 m} d \omega$, where $\omega_{c} \approx m_{e} c^{2}$ is the uv cutoff frequency with $m_{e}$ as the rest mass of electron, which depends on the kinetic energy of the free electron. The kinetic energy of the electron at the atomic state $|i\rangle$ is $\frac{\left\langle i\left|\mathbf{p}^{2}\right| i\right\rangle}{2 m}$. Therefore, the self-energy of the electron at state $|i\rangle$ can be written as

$$
\begin{aligned}
E_{s e} & =-\frac{1}{4 \pi \varepsilon_{0}} \frac{2 e^{2}}{3 \pi m^{2} c^{3}} \int_{0}^{\omega_{c}}\left\langle i\left|\mathbf{p}^{2}\right| i\right\rangle d \omega \\
& =-\frac{2 \alpha}{3 \pi m^{2} c^{2}} \int_{0}^{\omega_{c}} d \omega \sum_{j}\left|\mathbf{p}_{i j}\right|^{2} \\
& =-\sum_{j \neq i, \mathbf{k}} \frac{g_{\mathbf{k}, i j}^{2}}{\omega_{k}},
\end{aligned}
$$

where $\alpha=\frac{e^{2}}{4 \pi c \varepsilon_{0}}$ is the fine structure constant. In the last step, we changed the integration to the summation via $\frac{2 \alpha}{3 \pi(m c)^{2}} p_{i j}^{2} \int_{0}^{\infty} d \omega_{k} h\left(\omega_{k}\right)=\sum_{\mathbf{k}} \frac{g_{\mathbf{k}, i j}^{2}}{\omega_{k}} h\left(\omega_{k}\right)[2]$ where $h(\omega)$ is an arbitrary function. It follows, on subtracting the self-energy from $H_{2}^{S}$, for the mass renormalization, we have

$$
H_{2}^{S}-E_{s e}=-\sum_{i, j, \mathbf{k}}^{i \neq j} \frac{g_{\mathbf{k}, i j}^{2}}{\omega_{k}}\left[2 \xi_{k, i j}-\xi_{k, i j}^{2}-1-\frac{\xi_{k, i j}^{2}}{\omega_{k}}\left(\omega_{j}-\omega_{i}\right)\right]|i\rangle\langle i| .
$$

We can separate the transformed Hamiltonian,

$$
H^{S} \approx H_{0}^{S}+H_{1}^{S}
$$

into an off-diagonal part $H_{1}^{S}$ [Eq. (9)] and a diagonal part $H_{0}^{S}$,

$$
H_{0}^{S}=H_{0}+H_{2}^{S}-E_{s e}=\sum_{i} \omega_{i}^{\prime}|i\rangle\langle i|+\sum_{\mathbf{k}} \omega_{k} b_{\mathbf{k}}^{\dagger} b_{\mathbf{k}},
$$

with the new eigenfrequency $\omega_{i}^{\prime}=\omega_{i}+\Delta E_{n d}^{(i)}$, with 


$$
\begin{aligned}
& \Delta E_{n d}^{(i)}=\sum_{\mathbf{k}} \sum_{j<i} \frac{2 g_{\mathbf{k}, i j}^{2}}{\omega_{k}} \frac{\omega_{i j}^{2}}{\left(\omega_{k}+\left|\omega_{i j}\right|\right)^{2}}+\sum_{\mathbf{k}} \sum_{j} \frac{g_{\mathbf{k}, i j}^{2}}{\omega_{k}} \frac{\omega_{j i}}{\left(\omega_{k}+\left|\omega_{i j}\right|\right)} \\
& =\sum_{\mathbf{k}} \sum_{j<i} \frac{g_{\mathbf{k}, i j}^{2}}{\omega_{k}} \frac{\left(\omega_{i j}^{2}-\omega_{i j} \omega_{k}\right)}{\left(\omega_{k}+\omega_{i j}\right)^{2}}+\sum_{\mathbf{k}} \sum_{j>i} \frac{g_{\mathbf{k}, i j}^{2}}{\omega_{k}} \frac{\omega_{j i}}{\left(\omega_{k}+\omega_{j i}\right)} \\
& =\sum_{\mathbf{k}} \sum_{j \neq i} \frac{g_{\mathbf{k}, i j}^{2}}{\omega_{k}} \frac{\omega_{j i}\left(\omega_{j i}+\omega_{k}\right)}{\left(\omega_{k}+\mid \omega_{i j}\right)^{2}} .
\end{aligned}
$$

Due to the renormalization, the energy of the atom is shifted from $\omega_{i}$ to $\omega_{i}^{\prime}$ by an amount $\Delta E_{n d}^{(i)}$. This is the so-called nondynamic shift as it is not related to any decay process. We note that the effects of the counter-rotating terms are included in the new coupling constant and the new eigenfrequencies. The Hamiltonian [Eq. (13)] is in the RWA form and is valid for any multilevel quantum system.

\section{GROUND STATE}

Before studying the dynamic evolution of the atom, we consider the ground states of the interaction picture Hamiltonian $H_{0}^{S}=H_{0}+H_{2}^{S}-E_{s e}$, where $E_{s e}$ is the self-energy of the free electron. The ground state of $H_{0}^{S}$ is $\left|g^{S}\right\rangle=|g\rangle\left|\left\{0_{\mathbf{k}}\right\}\right\rangle$ with the eigenenergy $\omega_{g}^{\prime}=\omega_{g}+\left\langle\left\{0_{\mathbf{k}}\right\}\left|\left\langle g\left|H_{2}^{S}-E_{s e}\right| g\right\rangle\right|\left\{0_{\mathbf{k}}\right\}\right\rangle$, where $|g\rangle$ is the ground state of the bare atom $\left(H_{0}|g\rangle=\omega_{g}|g\rangle\right)$ and $\left|\left\{0_{k}\right\}\right\rangle$ is the vacuum state of the field. This ground state $\left|g^{S}\right\rangle$ $=|g\rangle\left|\left\{0_{\mathrm{k}}\right\}\right\rangle$ is also the ground state of the total Hamiltonian, $H^{S} \approx H_{0}^{S}+H_{1}^{S}$, because $H_{1}^{S}|g\rangle\left|\left\{0_{\mathbf{k}}\right\}\right\rangle=0$. The state $\left|\psi^{S}(t)\right\rangle$ in $H^{S}$ and the corresponding state $|\psi(t)\rangle$ in $H$ are related via $\left|\psi^{S}(t)\right\rangle=e^{i S \mid}|\psi(t)\rangle$. Therefore, the corresponding state of $\left|g^{S}\right\rangle$, for a two-level system (assuming $|e\rangle$ be the excited state of the atom with $\left.H_{0}|e\rangle=\omega_{e}|e\rangle\right)$, is

$$
\begin{aligned}
|\psi(t)\rangle= & e^{-i S \mid}\left|\psi^{S}(t)\right\rangle=|g\rangle\left|\left\{0_{\mathbf{k}}\right\}\right\rangle-\sum_{\mathbf{k}} \frac{g_{\mathbf{k}}}{\left(\omega_{\mathbf{k}}-\omega_{e g}\right)}|e\rangle\left|1_{\mathbf{k}}\right\rangle \\
& -\frac{1}{2} \sum_{\mathbf{k}} \frac{g_{\mathbf{k}}^{2}}{\left(\omega_{\mathbf{k}}-\omega_{e g}\right)^{2}}|g\rangle\left|\left\{0_{\mathbf{k}}\right\}\right\rangle,
\end{aligned}
$$

which is the same as in Ref. [13]. The state $|\psi(t)\rangle$ in $H$ can be expressed by the state $\left|\psi^{S}(t)\right\rangle$ in $H^{S}$ by $|\psi(t)\rangle=e^{-i S}\left|\psi^{S}(t)\right\rangle$. Therefore, the state $\left|g^{H}\right\rangle=|g\rangle\left|\left\{0_{\mathbf{k}}\right\}\right\rangle$ in $H$ with eigenenergy $\omega_{g}$ is not the ground state of the total system. If the atom initially is in $\left|g^{H}\right\rangle=|g\rangle\left|\left\{0_{\mathbf{k}}\right\}\right\rangle$, the atom will evolute some population to other states due to the counter-rotating terms.

\section{DYNAMIC EVOLUTION}

We consider an atom initially in the excited state $\left|e_{i}^{S}\right\rangle$ $=|i\rangle\left|\left\{0_{\mathbf{k}}\right\}\right\rangle$, which can be achieved by acting the Hermitian operator $(|i\rangle\langle g|+| g\rangle\langle i|)$ on the ground state. Here we calculate the time-dependent probability amplitude $\chi(t)$ $=\left\langle\left\{0_{\mathbf{k}}\right\}\left|\left\langle i\left|\exp \left(-i H^{S} t\right)\right| i\right\rangle\right|\left\{0_{\mathbf{k}}\right\}\right\rangle$.

The wave function can be written as

$$
|\varphi(t)\rangle=\chi(t)\left|i,\{0\}_{\mathbf{k}}\right\rangle+\sum_{j<i} \sum_{\mathbf{k}} \beta_{j, \mathbf{k}}(t)\left|j, 1_{\mathbf{k}}\right\rangle,
$$

where the state vector $\left|j, 1_{\mathbf{k}}\right\rangle$ represents the atom in the lower level $|j\rangle$ with the emission of a photon $\left|1_{\mathbf{k}}\right\rangle$. Applying Schrödinger equation to Eq. (16), we obtain

$$
\begin{gathered}
i \frac{d \chi(t)}{d t}=\sum_{j<i} \sum_{\mathbf{k}} \frac{2 \omega_{i j} g_{\mathbf{k}, i j} \xi_{k, i j}}{\omega_{k}} e^{i\left(\omega_{i j}^{\prime}-\omega_{k}\right) t} \beta_{j, \mathbf{k}}(t), \\
i \frac{d \beta_{j, \mathbf{k}}(t)}{d t}=\frac{2 \omega_{i j} g_{\mathbf{k}, i j} \xi_{k, i j}}{\omega_{k}} e^{-i\left(\omega_{i j}^{\prime}-\omega_{k}\right) t} \chi(t),
\end{gathered}
$$

where $\omega_{i j}^{\prime} \equiv \omega_{i}^{\prime}-\omega_{j}^{\prime}$. Integrating Eq. (18) and then substituting into Eq. (17), we get

$$
\frac{d \chi(t)}{d t}=-\sum_{j<i} \sum_{\mathbf{k}} \frac{4 \omega_{i j}^{2} g_{\mathbf{k}, i j}^{2} \xi_{k, i j}^{2}}{\omega_{k}^{2}} \int_{0}^{t} e^{i\left(\omega_{i j}^{\prime}-\omega_{k}\right)\left(t-t^{\prime}\right)} \chi\left(t^{\prime}\right) d t^{\prime} .
$$

This equation can be solved by formally integrating Eq. (19), iterating and keeping terms up to $g_{\mathbf{k}, i j}$. We then obtain

$$
\chi(t)=1-\int_{0}^{t}\left(t-t^{\prime}\right) \sum_{j<i} \sum_{\mathbf{k}} \frac{4 \omega_{i j g_{\mathbf{k}, i j}^{2}}^{2} \xi_{k, i j}^{2}}{\omega_{k}^{2}} e^{i\left(\omega_{i j}^{\prime}-\omega_{k}\right) t^{\prime}} d t^{\prime} .
$$

We can approximately write

$$
\begin{aligned}
\chi(t) & \approx \exp \left[-\int_{0}^{t}\left(t-t^{\prime}\right) \sum_{j<i} \sum_{\mathbf{k}} \frac{4 \omega_{i j}^{2} g_{\mathbf{k}, i j}^{2} \xi_{k, i j}^{2}}{\omega_{k}^{2}} e^{i\left(\omega_{i j}^{\prime}-\omega_{k}\right) t^{\prime}} d t^{\prime}\right] \\
& =\exp \left\{-t\left[-\frac{1}{t} \sum_{j<i} \sum_{\mathbf{k}} \frac{4 \omega_{i j}^{2} g_{\mathbf{k}, i j}^{2} \xi_{k, i j}^{2}}{\omega_{k}^{2}} \frac{e^{i\left(\omega_{i j}^{\prime}-\omega_{k}\right) t^{\prime}}-1-i\left(\omega_{i j}^{\prime}-\omega_{k}\right) t}{\left(\omega_{i j}^{\prime}-\omega_{k}\right)^{2}}\right]\right\} \\
& =\exp \left(-t\left[\sum_{j<i} \sum_{\mathbf{k}} \frac{4 \omega_{i j}^{2} g_{\mathbf{k}, i j}^{2} \xi_{k, i j}^{2}}{\omega_{k}^{2}} \frac{2 \sin ^{2}\left(\frac{\omega_{i j}^{\prime}-\omega_{k}}{2} t\right)}{\left(\omega_{i j}^{\prime}-\omega_{k}\right)^{2} t}+i \sum_{j<i} \sum_{\mathbf{k}} \frac{4 \omega_{i j}^{2} g_{\mathbf{k}, i j}^{2} \xi_{k, i j}^{2}}{\omega_{k}^{2}}\left[\frac{1}{\omega_{i j}^{\prime}-\omega_{k}}-\frac{\sin \left(\omega_{i j}^{\prime}-\omega_{k}\right) t}{\left(\omega_{i j}^{\prime}-\omega_{k}\right)^{2} t}\right]\right\}\right) \\
& =\exp \left\{-\left[\gamma_{i}(t) / 2+i \Delta E_{d y n}^{(i)}(t)\right] t\right\},
\end{aligned}
$$

where $\gamma_{i}(t)$ is the time-dependent decay rate (effective decay rate) and the second term (the imaginary part) is the timedependent dynamic energy shift due to the phase accumulation of the probability amplitude of the upper state during the dynamical evolution (decay). 


\section{EFFECTIVE DECAY RATE}

According to Eq. (21), the survival population in the initial state is

$$
P(t)=|\chi(t)|^{2}=\exp \left[-\gamma_{i}(t) t\right] .
$$

Here $\gamma_{i}(t)$ is the effective decay rate

$$
\begin{aligned}
\gamma_{i}(t)= & 2 \pi \sum_{j<i} \sum_{\mathbf{k}} \frac{4 \omega_{i j}^{2} g_{\mathbf{k}, i j}^{2} \xi_{k, i j}^{2}}{\omega_{k}^{2}} \frac{2 \sin ^{2}\left(\frac{\omega_{i j}^{\prime}-\omega_{k}}{2} t\right)}{\pi\left(\omega_{i j}^{\prime}-\omega_{k}\right)^{2} t} \\
= & 2 \pi \int_{0}^{\infty} \sum_{j<i} \sum_{\mathbf{k}} \frac{4 \omega_{i j}^{2} g_{\mathbf{k}, i j}^{2} \xi_{k, i j}^{2}}{\omega_{k}^{2}} \delta\left(\omega-\omega_{k}\right) \\
& \times \frac{2 \sin ^{2}\left(\frac{\omega_{i j}^{\prime}-\omega}{2} t\right)}{\pi\left(\omega_{i j}^{\prime}-\omega\right)^{2} t} d \omega \\
= & 2 \pi \int_{0}^{\infty} \sum_{j<i} G_{i j}(\omega) f_{i j}(\omega) F\left(\omega-\omega_{i j}^{\prime}, t\right) d \omega \\
\approx & 2 \pi \int_{0}^{\infty} \sum_{j<i} G_{i j}^{\prime}(\omega) F\left(\omega-\omega_{i j}, t\right) d \omega,
\end{aligned}
$$

where $G_{i j}(\omega)=\Sigma_{\mathbf{k}} g_{\mathbf{k}, i j}^{2} \delta\left(\omega-\omega_{k}\right)$ as given in Eq. (4) and

$$
\begin{gathered}
G_{i j}^{\prime}(\omega)=\frac{4 G_{i j}(\omega)\left(\omega_{i j}\right)^{2}}{\left(\omega+\omega_{i j}\right)^{2}}=G_{i j}(\omega) f_{i j}(\omega), \\
F\left(\omega-\omega_{i j}, t\right)=\frac{2 \sin ^{2}\left(\frac{\omega_{i j}-\omega}{2} t\right)}{\pi\left(\omega_{i j}-\omega\right)^{2} t} .
\end{gathered}
$$

Here we replaced $\omega_{i j}^{\prime}$ by $\omega_{i j}$ as their difference is of the order $g_{\mathbf{k}}^{2}$.

It follows, on comparing Eq. (24) with the results under RWA $[2,3,13]$, i.e., $\gamma_{i, R W A}(t)=2 \pi \int_{0}^{\infty} \Sigma_{j<i} G_{i j}(\omega) F(\omega$ $\left.-\omega_{i j}, t\right) d \omega$, we find the counter-rotating terms are effectively taken into account by modulating the spectrum $G_{i j}(\omega)$ with multiplying a function [1]

$$
f_{i j}(\omega)=\left[1-\left(\omega-\omega_{i j}\right) /\left(\omega+\omega_{i j}\right)\right]^{2} .
$$

Note here $\omega_{i j}=\left|\omega_{i j}\right|$. The second term in Eq. (27) comes from the counter-rotating terms: it is proportional to $1 /\left(\omega+\omega_{i j}\right)$ and is zero at $\omega=\omega_{i j}$. For $\omega>\omega_{i j}, f_{i j}(\omega)<1$, and for $\omega$ $\gg \omega_{i j}, f_{i j}(\omega) \sim 0$, which means the higher frequency part of the interacting spectrum is greatly suppressed by the counterrotating terms. However it is important to note that the contributions of the counter-rotating terms turn to be important only for very short time, because the function $F\left(\omega-\omega_{i j}, t\right)$ becomes $\delta\left(\omega-\omega_{i j}\right)$ in the long-time limit, and we have the decay rate $\gamma(\infty)=\gamma_{R W A}(\infty)=2 \pi \sum_{j<i} G_{i j}\left(\omega_{i j}\right)$, the decay rate under the Weisskopf-Wigner approximation.

Next we consider the dynamic evolution of the decay process. Here we adopt the commonly used spectrum for the free vacuum, $G_{i j}(\omega)=\frac{a_{i j} \omega / 2}{\left[1+\left(\omega / \omega_{c}\right)^{2}\right]^{4}}$, as given in Refs. [1,8-10], where $a_{i j}$ is the coupling strength (a dimensionless constant).
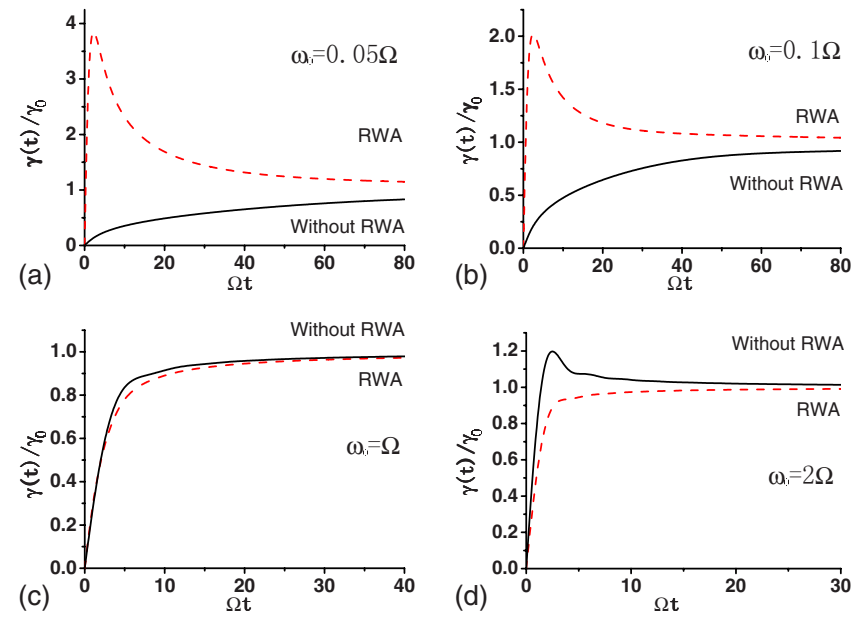

FIG. 1. (Color online) The effective decay rate $\gamma(t) / \gamma_{0}$ for different $\omega_{0}$.

This spectrum has a peak at $\Omega=\omega_{c} / \sqrt{7}$. We note that the spectrum function in Eq. (25) is modified from $G_{i j}(\omega)$ to $G_{i j}^{\prime}(\omega)=G_{i j}(\omega) \frac{4 \omega_{i j}^{2}}{\left(\omega_{i j}+\omega\right)^{2}}$. We consider the transition from the first excited state $(|e\rangle)$ to the ground state $(|g\rangle)$ with transition frequency $\omega_{i j}=\omega_{e}-\omega_{g}=\omega_{0}$, such as $2 P$ to $1 S$ of hydrogen atom. In Fig. 1, we plot the effective decay rate $\gamma(t) / \gamma_{0}$ for different $\omega_{0}$ [where $\gamma_{0}=2 \pi G_{e g}\left(\omega_{0}\right)$ is the decay rate for $|e\rangle$ $\rightarrow|g\rangle$ for a long time]. For comparison, we also plot $\gamma_{R W A}(t) / \gamma_{0}$ shown as the dotted line. It is known that for the hydrogen atom $\omega_{0} \ll \omega_{c}$, we have only the Zeno effect [1], see Fig. 1(a). For $\omega_{0}<\Omega$, the Zeno effect always dominates and there is no anti-Zeno effect for $\omega_{0}$ below $\Omega$. With the transition frequency increasing to the peak of the spectrum $(\Omega)$, the result without RWA is closer to the result of RWA. When the transition frequency is larger than $\Omega$, the anti-Zeno effect will be easier to realize as compared to the result from RWA.

The difference between the result with and without the RWA comes from the two counter-rotating terms $b_{\mathbf{k}}^{\dagger} e^{i \omega_{k} t}\left(|e\rangle\langle g| e^{i \omega_{0} t}\right)$ and $b_{\mathbf{k}} e^{-i \omega_{k} t}\left(|g\rangle\langle e| e^{-i \omega_{0} t}\right)$. For $\Omega \gg \omega_{0}$, the main part of the spectrum is in the region of $\omega_{k} \gg \omega_{0}$, and we have $b_{\mathbf{k}}^{\dagger} e^{i \omega_{k} t}\left(|e\rangle\langle g| e^{i \omega_{0} t}\right) \approx b_{\mathbf{k}}^{\dagger} e^{i \omega_{k} t}(|e\rangle\langle g|)$, whose role is just opposite to the role of the rotating term that can be written as $b_{\mathbf{k}}^{\dagger} e^{i \omega_{k} t}\left(|g\rangle\langle e| e^{-i \omega_{0} t}\right) \approx b_{\mathbf{k}}^{\dagger} e^{i \omega_{k} t}(|g\rangle\langle e|)$. Consequently, the counter-rotating term slows down the decay so that the Zeno effect dominates. For $\Omega \ll \omega_{0}$, the main part of the spectrum is in the region of $\omega_{k} \ll \omega_{0}$, and we have $b_{\mathbf{k}} e^{-i \omega_{k} t}\left(|g\rangle\langle e| e^{-i \omega_{0} t}\right) \approx b_{\mathbf{k}}\left(|g\rangle\langle e| e^{-i \omega_{0} t}\right)$, whose role is also making a decay, the same as the rotating terms $b_{\mathbf{k}}^{\dagger} e^{i \omega_{k} t}\left(|g\rangle\langle e| e^{-i \omega_{0} t}\right) \approx b_{\mathbf{k}}^{\dagger}\left(|g\rangle\langle e| e^{-i \omega_{0} t}\right)$. Consequently, the counter-rotating term accelerates the decay and anti-Zeno effect dominates. The role of the counter-rotating terms are well represented by the factor, $f\left(\omega_{k}\right)=\left[1-\left(\omega_{k}-\omega_{0}\right) /\left(\omega_{k}\right.\right.$ $\left.\left.+\omega_{0}\right)\right]^{2}$. For $\omega_{k} \gg \omega_{0}$, we have $f\left(\omega_{k}\right) \rightarrow 0$, slowing the decay (Zeno effect), while for $\omega_{k} \ll \omega_{0}$, we have $f\left(\omega_{k}\right) \rightarrow 4$, accelerating the decay (anti-Zeno).

In the above, we change the transition frequency while keeping the same spectrum. A similar result will be obtained if we change the spectrum while keeping the transition frequency constant. In general, there is no anti-Zeno effect but 
only Zeno effect when the transition frequency is smaller than the center frequency of the spectrum. When the transition frequency is larger than the center frequency of the spectrum, the anti-Zeno effect dominates. In the case that the transition frequency is near the center frequency of the spectrum, the counter-rotating terms seem to have no influence due to the opposite roles of the two parts of the high frequency and the low frequency in the spectrum. The quantum Zeno effect and quantum anti-Zeno effect will be found within the time scale of $10 / \Omega \sim 100 / \Omega$ with $\Omega$ being the spectrum center of the reservoir. This time scale also depends on the detailed spectrum and the atomic transition frequency.

\section{LAMB SHIFT}

The time-dependent dynamic shift for state $|i\rangle$, the second term in Eq. (21), is

$$
\begin{aligned}
\Delta E_{d y n}^{(i)}(t) & =\sum_{j<i} \sum_{\mathbf{k}} \frac{4 g_{\mathbf{k}, i j}^{2} \omega_{i j}^{2}}{\left(\omega_{i j}+\omega_{k}\right)^{2}}\left[\frac{1}{\omega_{i j}^{\prime}-\omega_{k}}-\frac{\sin \left(\omega_{i j}^{\prime}-\omega_{k}\right) t}{\left(\omega_{i j}^{\prime}-\omega_{k}\right)^{2} t}\right] \\
& =\sum_{j<i} \sum_{\mathbf{k}} \frac{4 g_{\mathbf{k}, i j}^{2} \omega_{i j}^{2}}{\left(\omega_{i j}+\omega_{k}\right)^{2}} \frac{1}{\omega_{i j}-\omega_{k}}\left[1-\frac{\sin \left(\omega_{i j}-\omega_{k}\right) t}{\left(\omega_{i j}-\omega_{k}\right) t}\right],
\end{aligned}
$$

where $\omega_{i}^{\prime}=\omega_{i}+\Delta E_{n d}$ taking into account the nondynamic level shift. However, the difference between $\omega_{i}^{\prime}$ and $\omega_{i}$ is on the order of $g^{2}$ and then $\omega_{i}^{\prime}$ can be replaced by $\omega_{i}$ in Eq. (28). The total energy shift at time $t$ is the sum of the nondynamic shift, Eq. (14b), and the dynamic shift [Eq. (28)],

$$
\begin{aligned}
\Delta E_{\text {total }}^{(i)}(t)= & \Delta E_{d y n}^{(i)}(t)+\Delta E_{n d}^{(i)} \\
= & \sum_{\mathbf{k}} \sum_{j<i} \frac{4 \omega_{i j}^{2}}{\left(\omega_{i j}+\omega_{k}\right)^{2}} \frac{g_{\mathbf{k}, i j}^{2}}{\omega_{i j}-\omega_{k}}\left[1-\frac{\sin \left(\omega_{i j}-\omega_{k}\right) t}{\left(\omega_{i j}-\omega_{k}\right) t}\right] \\
& +\sum_{\mathbf{k}} \sum_{j \neq i} \frac{g_{\mathbf{k}, i j}^{2}}{\omega_{k}} \frac{\omega_{j i}\left(\omega_{j i}+\omega_{k}\right)}{\left(\omega_{k}+\left|\omega_{i j}\right|\right)^{2}} .
\end{aligned}
$$

At the long-time limit, $\Delta E_{\text {total }}(\infty)$ can be written as (for details see Appendix C)

$$
\Delta E_{\text {total }}^{(i)}(\infty)=\Delta E_{d y n}^{(i)}(\infty)+\Delta E_{n d}^{(i)}=\sum_{\mathbf{k}} \sum_{j \neq i} \frac{g_{\mathbf{k}, i j}^{2}}{\omega_{k}} \frac{\omega_{i j}}{\left(\omega_{i j}-\omega_{k}\right)},
$$

which is the same as in [2], but different from that in [3].

\section{SPECTRUM OF THE FIELD EMITTED BY THE ATOM}

Considering the transition from the first excited state $|e\rangle$ to the ground state $|g\rangle$ with the transition frequency $\omega_{0}^{\prime}$ in $S$ picture, the equation for the probability amplitude of the ground state, $\beta_{\mathbf{k}}(t)$ (set $i=e, \quad j=g$ ) becomes $i \frac{d \beta_{\mathbf{k}}(t)}{d t}$ $=\frac{2 \omega_{0} g_{\mathbf{k}}}{\omega_{0}+\omega_{k}} e^{-i\left(\omega_{0}^{\prime}-\omega_{k}\right) t} \chi(t)$, see Eq. (18), where $\omega_{i j}^{\prime}=\omega_{e g}^{\prime}=\omega_{0}^{\prime}$. Substituting Eq. (21) into Eq. (18) and taking the long-time limit, we have

$$
\beta_{\mathbf{k}}(\infty)=\frac{g_{k}}{\left(\omega_{0}^{\prime}+\Delta E_{d y n}^{(e)}-\omega_{k}\right)+i \gamma_{0} / 2} \frac{2 \omega_{0}}{\left(\omega_{k}+\omega_{0}\right)},
$$

where $\gamma_{0}=\frac{\omega_{0}}{3 \pi \varepsilon_{0} c^{3}}\left(\frac{e}{m}\left|p_{e g}\right|\right)^{2}$ is the decay rate under the WignerWeisskopf approximation [15]. The spectral of the field emitted by the atom is

$$
\begin{aligned}
\left\langle\varphi(t)\left|E^{-}(t) E^{+}(t)\right| \varphi(t)\right\rangle_{t=\infty} & =\left\langle\varphi(t)\left|\sum_{\mathbf{k}, \mathbf{k}^{\prime}} b_{\mathbf{k}}^{\dagger} b_{\mathbf{k}^{\prime}} e^{i \omega_{k} t} e^{-i \omega_{k^{\prime}} t}\right| \varphi(t)\right\rangle_{t=\infty} \\
& =\sum_{\mathbf{k}} \beta_{\mathbf{k}}^{*}(\infty) \beta_{\mathbf{k}}(\infty) \\
& =\int_{-\infty}^{\infty} D\left(\omega_{k}\right) \frac{\beta_{\mathbf{k}}^{*}(\infty) \beta_{\mathbf{k}}(\infty)}{g_{\mathbf{k}}^{2}} d \omega_{k} \\
& =\int d \omega_{k} S\left(\omega_{k}\right),
\end{aligned}
$$

where $E^{-}(t)$ and $E^{+}(t)$ are the electric field operators, $g_{\mathbf{k}}^{2}$ $=\left[\frac{1}{2 \varepsilon_{0} L^{3}}\left(\frac{e}{m} \hat{\mathbf{e}}_{\mathbf{k}} \cdot \mathbf{p}_{i j}\right)^{2}\right] \frac{1}{\omega_{k}}$ and $D\left(\omega_{k}\right)=\frac{\omega_{k}}{6 \pi^{2} \varepsilon_{0} c^{3}}\left(\frac{e}{m}\left|\mathbf{p}_{e g}\right|\right)^{2}$. Using Eq. (31) we have

$$
\begin{aligned}
S\left(\omega_{k}\right)= & \frac{\omega_{k}}{6 \pi^{2} \varepsilon_{0} c^{3}}\left(\frac{e}{m}\left|\mathbf{p}_{e g}\right|\right)^{2} \\
& \times \frac{1}{\left(\omega_{0}^{\prime}+\Delta E_{d y n}^{(e)}-\omega_{k}\right)^{2}+\gamma_{0}^{2} / 4} \frac{4 \omega_{0}^{2}}{\left(\omega_{k}+\omega_{0}\right)^{2}} \\
= & \frac{\omega_{k}}{\pi \omega_{0}} \frac{\gamma_{0} / 2}{\left[\left(\omega_{0}^{\prime}+\Delta E_{d y n}^{(e)}\right)-\omega_{k}\right]^{2}+\gamma_{0}^{2} / 4} \frac{4 \omega_{0}^{2}}{\left(\omega_{k}+\omega_{0}\right)^{2}}, \\
= & \frac{\omega_{k}}{\pi \omega_{0}} \frac{\gamma_{0} / 2}{\left[\left(\omega_{0}+\Delta E_{n d}^{(e)}-\Delta E_{n d}^{(g)}+\Delta E_{d y n}^{(e)}\right)-\omega_{k}\right]^{2}+\gamma_{0}^{2} / 4} \\
=\frac{4 \omega_{0}^{2}}{\pi \omega_{0}} & \frac{\omega_{0} / 2}{\left(\omega_{k}+\omega_{0}\right)^{2}},
\end{aligned}
$$

where the energy shift for the first excited state $|e\rangle$ is $\Delta E_{\text {total }}^{(e)}=\Delta E_{n d}^{(e)}+\Delta E_{d y n}^{(e)}$. The spectral obtained from previous methods, such as in [2], is

$$
S_{\text {pre }}\left(\omega_{k}\right)=\frac{\omega_{k}}{\pi \omega_{0}} \frac{\gamma_{0} / 2}{\left[\left(\omega_{0}+\Delta E_{\text {total }}^{(e)}\right)-\omega_{k}\right]^{2}+\gamma_{0}^{2} / 4} .
$$

Please note two differences, the term of $-\Delta E_{n d}^{(g)}$ in the denominator, which leads to different peak positions, and the additional factor of $f\left(\omega_{k}\right)=4 \omega_{0}^{2} /\left(\omega_{k}+\omega_{0}\right)^{2}$, which leads to a shift of the distribution toward the low frequency region, because of $f\left(\omega_{k}<\omega_{0}\right)>1$, and $f\left(\omega_{k}>\omega_{0}\right)<1$.

In Fig. 2, we plot the spectral distribution for the $2 P_{1 / 2}$ state of the hydrogen atom, $\Delta E_{\text {total }}^{(e)}=0, \gamma_{2 P} \approx 4.04 \times 10^{-8} \omega_{P S}$ $=1.01 \times 10^{8} \mathrm{~Hz} \quad\left(\omega_{P S} \approx 1.55 \times 10^{16} \mathrm{rad} / \mathrm{s}=2.5 \times 10^{15} \mathrm{~Hz}\right)$, and $\Delta E_{n d}^{(g)} \approx 8 \times 10^{9} \mathrm{~Hz}[16]$, the solid line for Eq. (33) and dashed line for Eq. (34). Someone may argue that the shift of $-\Delta E_{n d}^{(g)}$ can be included in the previous methods, as eventually the atom will decay to the ground state. However, in the 


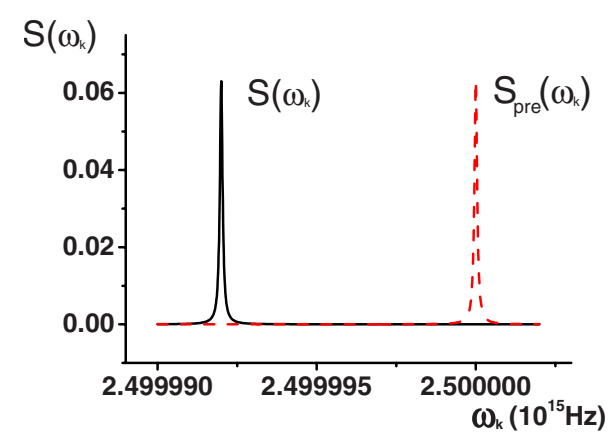

FIG. 2. (Color online) The spectrum $S\left(\omega_{k}\right)$ in arbitrary unit for the hydrogen transition from $2 P$ to $1 S$ state.

previous method, the energy shift of the ground state is also accompanied by the transition to upper states due to the counter-rotating terms, which results in zero decay rate at the long-time limit but not zero population in the upper states. The key problem in the previous method is that the bare ground state of the atom with no photon is not the ground state of the whole system as we discussed in Sec. III. If we add the energy shift of the ground state by hand in the previous methods, Eq. (34) can be revised as

$$
S_{\text {pre }}^{\prime}\left(\omega_{k}\right)=\frac{\omega_{k}}{\pi \omega_{0}} \frac{\gamma_{0} / 2}{\left[\left(\omega_{0}+\Delta E_{\text {total }}^{(e)}-\Delta E_{n d}^{(g)}\right)-\omega_{k}\right]^{2}+\gamma_{0}^{2} / 4} .
$$

The difference in the denominator will be eliminated. The energy shift of the ground state is the contribution of the counter-rotating terms and the nondynamical shift $\Delta E_{n d}^{(g)}$ is just the Lamb shift $\left(1 S_{1 / 2}\right)$, which is self-consistently included in our method without manual correction. The difference (about $8 \times 10^{9} \mathrm{~Hz}$ ) between the peaks between $S\left(\omega_{k}\right)$ and $S_{\text {pre }}\left(\omega_{k}\right)$ is achievable by current technology.

\section{SUMMARY}

We presented a method based on a unitary transformation that can take into account the counter-rotating as well as the rotating terms in the Hamiltonian. By using the ground state of the total Hamiltonian, we have derived the effective decay rate and the Lamb shift without the RWA. The counterrotating terms have important contribution to the short-time dynamic evolution in the atomic decay. If the atomic transition frequency is smaller than the central frequency of the spectrum, the Zeno effect dominates, and if the atomic transition frequency is larger than the central frequency of the spectrum, the anti-Zeno effect will dominate. The time evolution of the energy shift is also obtained. The statistical frequency distribution of the photon emitted by the atom is shifted to the low frequency region compared with the same distribution obtained under the rotating wave approximation.

\section{ACKNOWLEDGMENTS}

This work was supported by RGC of Hong Kong Government and FRG of Hong Kong Baptist University. The re- search of M.S.Z. was supported by a grant from the Qatar National Research Fund (QNRF).

\section{APPENDIX A: THE CORRECT GROUND STATE OF THE WHOLE SYSTEM}

\section{Time evolution of the ground state of the bare atom with no photon, $\left|g^{H}\right\rangle=|g\rangle\left|\left\{0_{\mathrm{k}}\right\}\right\rangle$}

Consider a two-level system with ground state $|g\rangle$ and excited state $|e\rangle$ of the bare atom, respectively. After subtracting the self-energy of the free electron, $E_{S E_{-} i}$ $=-\sum_{\mathbf{k}} g_{\mathbf{k}}^{2} / \omega_{k}(i=g, e)$, the Hamiltonian in Schrödinger picture is

$$
\begin{aligned}
H= & \omega_{e}^{\prime}|e\rangle\left\langle e\left|+\omega_{g}^{\prime}\right| g\right\rangle\langle g|+\sum_{\mathbf{k}} \omega_{k} b_{\mathbf{k}}^{\dagger} b_{\mathbf{k}}+\sum_{\mathbf{k}} g_{k}\left(b_{\mathbf{k}}^{\dagger}+b_{\mathbf{k}}\right)(|e\rangle\langle g| \\
& +|g\rangle\langle e|),
\end{aligned}
$$

where $\omega_{i}^{\prime}=\omega_{i}-E_{S E_{-} i}$. Note $E_{S E_{-} i}$ is the same for $|e\rangle$ and $|g\rangle$ in the two-level system. With the wave function $|\varphi(t)\rangle$ $=\chi(t)\left|g,\left\{0_{\mathbf{k}}\right\}\right\rangle+\Sigma \beta_{k}(t)\left|e, 1_{\mathbf{k}}\right\rangle$ in the interaction picture and the $\mathbf{k}$

initial state $\varphi(0)=|g\rangle\left|\left\{0_{\mathbf{k}}\right\}\right\rangle$, we can obtain

$$
\begin{gathered}
i \frac{d \chi(t)}{d t}=\sum_{\mathbf{k}} g_{\mathbf{k}} e^{i\left(-\omega_{0}-\omega_{k}\right) t} \beta_{\mathbf{k}}(t), \\
i \frac{d \beta_{\mathbf{k}}(t)}{d t}=g_{\mathbf{k}} e^{-i\left(-\omega_{0}-\omega_{k}\right) t} \chi(t),
\end{gathered}
$$

where $\omega_{0}=\omega_{e}-\omega_{g}$. Integrating Eq. (A3) and then substituting it into Eq. (A2), we have

$$
\frac{d \chi(t)}{d t}=-\sum_{k} g_{\mathbf{k}}^{2} \int_{0}^{t} e^{i\left(-\omega_{0}-\omega_{k}\right)\left(t-t^{\prime}\right)} \chi\left(t^{\prime}\right) d t^{\prime} .
$$

Integrating Eq. (A4) and with the similar calculation in Sec. IV, we have

$$
\begin{aligned}
\chi(t)= & \chi(0) \exp \left(-t\left\{\sum_{\mathbf{k}} g_{\mathbf{k}}^{2} \frac{2 \sin ^{2}\left(\frac{\omega_{0}+\omega_{k}}{2} t\right)}{\left(\omega_{0}+\omega_{k}\right)^{2} t}\right.\right. \\
& \left.\left.+i \sum_{\mathbf{k}} g_{\mathbf{k}}^{2}\left[-\frac{1}{\omega_{0}+\omega_{k}}+\frac{\sin \left(\omega_{0}+\omega_{k}\right) t}{\left(\omega_{0}+\omega_{k}\right)^{2} t}\right]\right\}\right) .
\end{aligned}
$$

The survival population of the ground state $\left|g,\left\{0_{\mathbf{k}}\right\}\right\rangle$ is

$$
\begin{aligned}
P_{g}(t)= & |\chi(t)|^{2}=\exp \left(-2 \sum_{\mathbf{k}} g_{\mathbf{k}}^{2} \frac{2 \sin ^{2}\left(\frac{\omega_{0}+\omega_{k}}{2} t\right)}{\left(\omega_{0}+\omega_{k}\right)^{2}}\right) \underset{t \rightarrow \infty}{\approx}=1 \\
& -2 \sum_{\mathbf{k}} g_{\mathbf{k}}^{2} \frac{1}{\left(\omega_{0}+\omega_{k}\right)^{2}},
\end{aligned}
$$

In the last, $2 \sin ^{2}\left(\frac{\omega_{0}+\omega_{k}}{2} t\right)$ was replaced by its average value (equal to 1). In Fig. 3 we plot the evolution of the population, where we use the spectrum function $G(\omega)=\frac{a_{0} \omega / 2}{\left[1+\left(\omega / \omega_{c}\right)^{2}\right]^{4}}$ with 


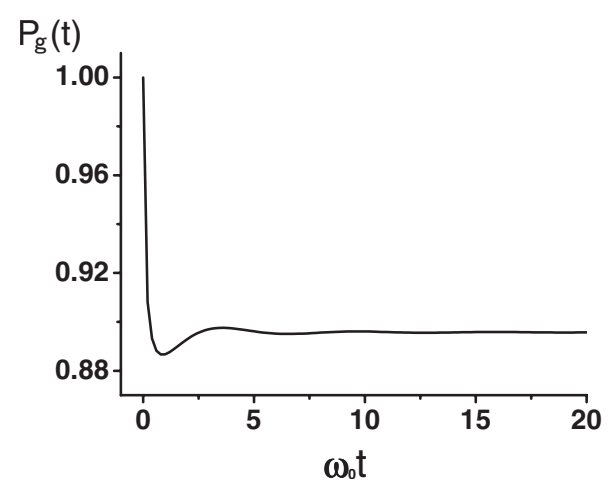

FIG. 3. The evolution of the survival population, $P_{g}(t)$.

the parameter $a_{0}=0.02$ and the cutoff frequency $\omega_{c}=100 \omega_{0}$. It is clear that $\left|g^{H}\right\rangle=|g\rangle\left|\left\{0_{\mathbf{k}}\right\}\right\rangle$ is not the ground state of the whole system, which is given in Sec. III, $\left|g^{S}\right\rangle$ in $S$ picture. The ground state of the whole system in the $H$ picture can be obtained by the unitary transformation,

$$
\begin{aligned}
|G\rangle= & e^{-i S \mid}\left|g^{S}\right\rangle \approx\left[1-i S-S^{2}\right]\left|g,\left\{0_{\mathbf{k}}\right\}\right\rangle \\
\approx & {\left[1-\frac{1}{2} \sum_{\mathbf{k}} \frac{g_{\mathbf{k}}^{2}}{\left(\omega_{\mathbf{k}}+\omega_{0}\right)^{2}}\right]\left|g,\left\{0_{\mathbf{k}}\right\}\right\rangle } \\
& -\sum_{\mathbf{k}} \frac{g_{\mathbf{k}}}{\left(\omega_{\mathbf{k}}+\omega_{0}\right)}\left|e, 1_{\mathbf{k}}\right\rangle,
\end{aligned}
$$

where the two-photon terms are dropped. It is not difficult to find that there is no population evolution if the system initially is in the $|G\rangle$ state, except a phase depending on the energy of the $|G\rangle$ state.

\section{Energies of the $\left|g,\left\{0_{\mathrm{k}}\right\}\right\rangle$ state and the $|G\rangle$ state}

The energy of $\left|g,\left\{0_{\mathbf{k}}\right\}\right\rangle$ is

$$
\left\langle g,\left\{0_{\mathbf{k}}\right\}|H| g,\left\{0_{\mathbf{k}}\right\}\right\rangle=\omega_{g}^{\prime}=\omega_{g}+\sum_{\mathbf{k}} \frac{g_{\mathbf{k}}^{2}}{\omega_{k}} .
$$

The energy of $|G\rangle$ is

$$
\begin{aligned}
\langle G|H| G\rangle & =\left\langle G\left|\left\{\omega_{e}^{\prime}|e\rangle\left\langle e\left|+\omega_{g}^{\prime}\right| g\right\rangle\langle g|+\sum_{\mathbf{k}} \omega_{k} b_{\mathbf{k}}^{\dagger} b_{\mathbf{k}}+\sum_{\mathbf{k}} g_{\mathbf{k}}\left(b_{\mathbf{k}}^{\dagger}+b_{\mathbf{k}}\right)(|e\rangle\langle g|+| g\rangle\langle e|)\right\}\right| G\right\rangle \\
& =\left\langle G\left|\left[\omega_{e}^{\prime}|e\rangle\left\langle e\left|+\omega_{g}^{\prime}\right| g\right\rangle\langle g|+\sum_{\mathbf{k}} \omega_{\mathbf{k}} b_{\mathbf{k}}^{\dagger} b_{\mathbf{k}}\right]\right| G\right\rangle+\left\langle G\left|\sum_{\mathbf{k}} g_{\mathbf{k}}\left(b_{\mathbf{k}}^{\dagger}+b_{\mathbf{k}}\right)(|e\rangle\langle g|+| g\rangle\langle e|)\right| G\right\rangle \\
& =(\mathrm{I})+(\mathrm{II})=\omega_{g}+\sum_{\mathbf{k}} \frac{g_{\mathbf{k}}^{2}}{\omega_{k}}-\sum_{k} \frac{g_{\mathbf{k}}^{2}}{\left(\omega_{k}+\omega_{0}\right)}=\omega_{g}+\sum_{\mathbf{k}} \frac{g_{\mathbf{k}}^{2} \omega_{0}}{\omega_{k}\left(\omega_{k}+\omega_{0}\right)},
\end{aligned}
$$

where

$$
\begin{aligned}
&(\mathrm{I})= \omega_{e}^{\prime} \sum_{\mathbf{k}} \frac{g_{\mathbf{k}}^{2}}{\left(\omega_{\mathbf{k}}+\omega_{0}\right)^{2}}+\sum_{\mathbf{k}} \frac{\omega_{k} g_{\mathbf{k}}^{2}}{\left(\omega_{k}+\omega_{0}\right)^{2}}+\omega_{g}^{\prime}\left[1-\sum_{\mathbf{k}} \frac{1}{2} \frac{g_{\mathbf{k}}^{2}}{\left(\omega_{k}+\omega_{0}\right)^{2}}\right]^{2} \\
& \approx \omega_{e}^{\prime} \sum_{\mathbf{k}} \frac{g_{\mathbf{k}}^{2}}{\left(\omega_{k}+\omega_{0}\right)^{2}}+\sum_{\mathbf{k}} \frac{\omega_{k} g_{\mathbf{k}}^{2}}{\left(\omega_{k}+\omega_{0}\right)^{2}}+\omega_{g}^{\prime}\left[1-\sum_{\mathbf{k}} \frac{g_{\mathbf{k}}^{2}}{\left(\omega_{k}+\omega_{0}\right)^{2}}\right] \\
&=\omega_{g}+\sum_{\mathbf{k}} \frac{g_{\mathbf{k}}^{2}}{\omega_{k}}+\sum_{\mathbf{k}} \frac{g_{\mathbf{k}}^{2}}{\left(\omega_{k}+\omega_{0}\right)}, \\
&(\mathrm{II})=\left\langle G\left|\sum_{\mathbf{k}} g_{\mathbf{k}}\left(b_{\mathbf{k}}^{\dagger}|e\rangle\left\langle g\left|+b_{\mathbf{k}}^{\dagger}\right| g\right\rangle\left\langle e\left|+b_{\mathbf{k}}\right| e\right\rangle\left\langle g\left|+b_{\mathbf{k}}\right| g\right\rangle\langle e|\right)\right| G\right\rangle \\
&=\left\langle G\left|\sum_{\mathbf{k}} g_{\mathbf{k}}\left(b_{\mathbf{k}}^{\dagger}|e\rangle\left\langle g\left|+b_{\mathbf{k}}\right| g\right\rangle\langle e|\right)\right| G\right\rangle \\
&=-\sum_{\mathbf{k}} g_{\mathbf{k}} \frac{g_{\mathbf{k}}}{\left(\omega_{k}+\omega_{0}\right)}\left[1-\sum_{\mathbf{k}} \frac{1}{2} \frac{g_{\mathbf{k}}^{2}}{\left(\omega_{k}+\omega_{0}\right)^{2}}\right] \\
&-\sum_{\mathbf{k}} g_{\mathbf{k}}\left[1-\sum_{\mathbf{k}} \frac{1}{2} \frac{g_{\mathbf{k}}^{2}}{\left(\omega_{k}+\omega_{0}\right)^{2}}\right] \frac{g_{\mathbf{k}}}{\left(\omega_{k}+\omega_{0}\right)} \\
& \approx-\sum_{\mathbf{k}} \frac{2 g_{\mathbf{k}}^{2}}{\left(\omega_{k}+\omega_{0}\right)},
\end{aligned}
$$

Note the second term in Eq. (A9) is the nondynamical shift of the state $|g\rangle$ with the self-energy subtracted. The energy difference between the two states is $\left\langle g,\left\{0_{\mathbf{k}}\right\}|H| g,\left\{0_{\mathbf{k}}\right\}\right\rangle-\langle G|H| G\rangle=\sum_{\mathbf{k}} \frac{g_{\mathbf{k}}^{2}}{\omega_{k}+\omega_{0}}>0$. 


\section{Energies of two excited states}

The excited state can be achieved by acting $\sigma_{x}=(|e\rangle\langle g|$ $+|g\rangle\langle e|)$ on the ground state in $H$ picture. For the state $\left|g,\left\{0_{\mathbf{k}}\right\}\right\rangle$, which is $\left|e,\left\{0_{\mathbf{k}}\right\}\right\rangle$. Its energy is

$$
\left\langle e,\left\{0_{\mathbf{k}}\right\}|H| e,\left\{0_{\mathbf{k}}\right\}\right\rangle=\omega_{e}^{\prime}=\omega_{e}+\sum_{\mathbf{k}} \frac{g_{\mathbf{k}}^{2}}{\omega_{k}} .
$$

The other excited state is obtained by acting $\sigma_{x}^{S}=\sigma_{x}$ on the state $|G\rangle,\left|e^{S}\right\rangle=\sigma_{x}|G\rangle=\left|e,\left\{0_{\mathbf{k}}\right\}\right\rangle$ in the $S$ picture. This excited state in $H$ picture can be obtained:

$$
\begin{aligned}
|E\rangle= & e^{-i S}\left|e,\left\{0_{\mathbf{k}}\right\}\right\rangle \approx\left[1-i S-S^{2}\right]\left|e,\left\{0_{\mathbf{k}}\right\}\right\rangle \approx\left|e,\left\{0_{\mathbf{k}}\right\}\right\rangle \\
& -\sum_{\mathbf{k}} \frac{g_{\mathbf{k}}}{\left(\omega_{k}+\omega_{0}\right)}\left|g, 1_{\mathbf{k}}\right\rangle-\frac{1}{2} \sum_{\mathbf{k}} \frac{g_{\mathbf{k}}^{2}}{\left(\omega_{k}+\omega_{0}\right)^{2}}\left|e,\left\{0_{\mathbf{k}}\right\}\right\rangle .
\end{aligned}
$$

The energy of the state $|E\rangle$ is

$$
\begin{aligned}
\langle E|H| E\rangle & =\left\langle E\left|\left[\omega_{e}^{\prime}|e\rangle\left\langle e\left|+\omega_{g}^{\prime}\right| g\right\rangle\langle g|+\sum_{\mathbf{k}} \omega_{k} b_{\mathbf{k}}^{\dagger} b_{\mathbf{k}}\right]\right| E\right\rangle+\left\langle E\left|\sum_{\mathbf{k}} g_{\mathbf{k}}\left(b_{\mathbf{k}}^{\dagger}+b_{\mathbf{k}}\right)(|e\rangle\langle g|+| g\rangle\langle e|)\right| E\right\rangle=\left(\mathrm{I}^{\prime}\right)+\left(\mathrm{II}^{\prime}\right) \\
& =\omega_{e}^{\prime}+\sum_{\mathbf{k}} \frac{g_{\mathbf{k}}^{2}\left(\omega_{k}-\omega_{0}\right)}{\left(\omega_{k}+\omega_{0}\right)^{2}}-\sum_{\mathbf{k}} \frac{2 g_{\mathbf{k}}^{2}}{\left(\omega_{k}+\omega_{0}\right)}=\omega_{e}^{\prime}+\sum_{\mathbf{k}} \frac{g_{\mathbf{k}}^{2} \omega_{k}\left(-\omega_{k}-3 \omega_{0}\right)}{\omega_{k}\left(\omega_{k}+\omega_{0}\right)^{2}}=\omega_{e}^{\prime}+\sum_{\mathbf{k}} \frac{g_{\mathbf{k}}^{2} \omega_{0}\left(\omega_{0}-\omega_{k}\right)}{\omega_{k}\left(\omega_{k}+\omega_{0}\right)^{2}}-\sum_{\mathbf{k}} \frac{g_{\mathbf{k}}^{2}}{\omega_{k}},
\end{aligned}
$$

where

$$
\begin{gathered}
\left(\mathrm{I}^{\prime}\right)=\omega_{g}^{\prime} \sum_{\mathbf{k}} \frac{g_{\mathbf{k}}^{2}}{\left(\omega_{k}+\omega_{0}\right)^{2}}+\sum_{\mathbf{k}} \frac{\omega_{k} g_{\mathbf{k}}^{2}}{\left(\omega_{k}+\omega_{0}\right)^{2}}+\omega_{e}^{\prime}\left[1-\sum_{\mathbf{k}} \frac{1}{2} \frac{g_{\mathbf{k}}^{2}}{\left(\omega_{k}+\omega_{0}\right)^{2}}\right]^{2}=\omega_{e}^{\prime}+\sum_{\mathbf{k}} \frac{g_{\mathbf{k}}^{2}\left(\omega_{k}-\omega_{0}\right)}{\left(\omega_{k}+\omega_{0}\right)^{2}}, \\
\left(\mathrm{II}^{\prime}\right)=-\sum_{\mathbf{k}} g_{\mathbf{k}} \frac{g_{\mathbf{k}}}{\left(\omega_{k}+\omega_{0}\right)}\left[1-\sum_{\mathbf{k}} \frac{1}{2} \frac{g_{\mathbf{k}}^{2}}{\left(\omega_{k}+\omega_{0}\right)^{2}}\right]-\sum_{\mathbf{k}} g_{\mathbf{k}}\left[1-\sum_{\mathbf{k}} \frac{1}{2} \frac{g_{\mathbf{k}}^{2}}{\left(\omega_{k}+\omega_{0}\right)^{2}}\right] \frac{g_{\mathbf{k}}}{\left(\omega_{k}+\omega_{0}\right)} \approx-\sum_{\mathbf{k}} \frac{2 g_{\mathbf{k}}^{2}}{\left(\omega_{k}+\omega_{0}\right)} .
\end{gathered}
$$

Note the second term in Eq. (A12) is the nondynamical shift of the state $|e\rangle$ with the self-energy subtracted. The energy difference between the two states is $\left\langle e,\left\{0_{\mathbf{k}}\right\}|H| e,\left\{0_{\mathbf{k}}\right\}\right\rangle-\langle E|H| E\rangle=\Sigma_{\mathbf{k}} \frac{g_{\mathbf{k}}^{2} \omega_{k}\left(\omega_{k}+3 \omega_{0}\right)}{\omega_{k}\left(\omega_{k}+\omega_{0}\right)^{2}}>0$.

\section{APPENDIX B: THE CALCULATION OF $H_{2}^{S}$}

We recall that

$$
H_{2}^{S}=\left[i S, H_{1}\right]+\frac{1}{2}\left[i S,\left[i S, H_{0}\right]\right] .
$$

First, we calculate the commutation relation $\left[i S, H_{1}\right]$. It follows from Eqs. (3) and (5) that

$$
\begin{aligned}
{\left[i S, H_{1}\right] } & =\left[\sum_{i, j, \mathbf{k}}^{i \neq j} \frac{g_{\mathbf{k}, i j} \xi_{k, i j}}{\omega_{k}}\left(b_{\mathbf{k}}^{\dagger}-b_{\mathbf{k}}\right)|i\rangle\langle j|\right] \sum_{i, j, \mathbf{k}}^{i \neq j} g_{\mathbf{k}, i j}\left(b_{\mathbf{k}}^{\dagger}+b_{\mathbf{k}}\right)|i\rangle\left\langle j\left|-\left[\sum_{i, j, \mathbf{k}} g_{\mathbf{k}, i j}\left(b_{\mathbf{k}}^{\dagger}+b_{\mathbf{k}}\right)|i\rangle\langle j|\right] \sum_{i, j, \mathbf{k}}^{i \neq j} \frac{g_{\mathbf{k}, i j} \xi_{k, i j}}{\omega_{k}}\left(b_{\mathbf{k}}^{\dagger}-b_{\mathbf{k}}\right)\right| i\right\rangle\langle j| \\
& =\sum_{\mathbf{k}}\left[\left(b_{\mathbf{k}}^{\dagger}-b_{\mathbf{k}}\right)\left(b_{\mathbf{k}}^{\dagger}+b_{\mathbf{k}}\right)-\left(b_{\mathbf{k}}^{\dagger}+b_{\mathbf{k}}\right)\left(b_{\mathbf{k}}^{\dagger}-b_{\mathbf{k}}\right)\right] \sum_{i, j, j^{\prime}}^{i \neq j, j \neq j^{\prime}} \frac{g_{\mathbf{k}, i j} \xi_{k, i j}}{\omega_{k}} g_{\mathbf{k}, j j^{\prime}}|i\rangle\left\langle j^{\prime}\right|,
\end{aligned}
$$

which includes the diagonal as well as the off-diagonal terms. The diagonal terms will have the contribution to the energy shift, while the nondiagonal terms $\left(|i\rangle\left\langle j^{\prime}\right|\right.$ with $\left.i \neq j^{\prime}\right)$ are neglected as their contribution to the transition is of the fourth order in $g_{k}$. We note that $\left(b_{\mathbf{k}}^{\dagger}-b_{\mathbf{k}}\right)\left(b_{\mathbf{k}}^{\dagger}+b_{\mathbf{k}}\right)-\left(b_{\mathbf{k}}^{\dagger}+b_{\mathbf{k}}\right)\left(b_{\mathbf{k}}^{\dagger}-b_{\mathbf{k}}\right)=2\left(b_{\mathbf{k}}^{\dagger} b_{\mathbf{k}}-b_{\mathbf{k}} b_{\mathbf{k}}^{\dagger}\right)=-2$. Therefore, we have

$$
\left[i S, H_{1}\right]=2 \sum_{i, j, \mathbf{k}}^{i \neq j} \frac{g_{\mathbf{k}, i j}^{2} \xi_{k, i j}}{\omega_{k}}|i\rangle\langle i| .
$$

Next we consider the term $\frac{1}{2}\left[i S,\left[i S, H_{0}\right]\right]$. It follows from Eqs. (2) and $(5)$ that 


$$
\begin{aligned}
\frac{1}{2}\left[i S,\left[i S, H_{0}\right]\right]= & \frac{1}{2}\left\{\left[\sum_{i, j, \mathbf{k}}^{i \neq j} \frac{g_{\mathbf{k}, i j} \xi_{k, i j}}{\omega_{k}}\left(b_{\mathbf{k}}^{\dagger}-b_{\mathbf{k}}\right)|i\rangle\langle j|\right] \sum_{i, j \neq i, \mathbf{k}} g_{\mathbf{k}, i j} \xi_{k, i j}\left[\left(\omega_{j}-\omega_{i}\right)\left(b_{\mathbf{k}}^{\dagger}-b_{\mathbf{k}}\right) \frac{1}{\omega_{k}}-\left(b_{\mathbf{k}}^{\dagger}+b_{\mathbf{k}}\right)\right]|i\rangle\langle j|\right. \\
& \left.-\left\{\sum_{i, j \neq i, \mathbf{k}} g_{\mathbf{k}, i j} \xi_{k, i j}\left[\left(\omega_{j}-\omega_{i}\right)\left(b_{\mathbf{k}}^{\dagger}-b_{\mathbf{k}}\right) \frac{1}{\omega_{k}}-\left(b_{\mathbf{k}}^{\dagger}+b_{\mathbf{k}}\right)\right]|i\rangle\langle j|\right\} \sum_{i, j, \mathbf{k}}^{i \neq j} \frac{g_{\mathbf{k}, i j} \xi_{k, i j}}{\omega_{k}}\left(b_{\mathbf{k}}^{\dagger}-b_{\mathbf{k}}\right)|i\rangle\langle j|\right\} \\
= & \frac{1}{2}\left\{-\left[\sum_{i, j, \mathbf{k}}^{i \neq j} \frac{g_{\mathbf{k}, i j} \xi_{k, i j}}{\omega_{k}}\left(b_{\mathbf{k}}^{\dagger}-b_{\mathbf{k}}\right)|i\rangle\langle j|\right]\left[\sum_{i, j, \mathbf{k}}^{i \neq j} \frac{g_{\mathbf{k}, i j} \xi_{k, i j}}{\omega_{k}}\left(b_{\mathbf{k}}^{\dagger}+b_{\mathbf{k}}\right)|i\rangle\langle j|\right]+\left[\sum_{i, j, \mathbf{k}}^{i \neq j} \frac{g_{\mathbf{k}, i j} \xi_{k, i j}}{\omega_{k}}\left(b_{\mathbf{k}}^{\dagger}+b_{\mathbf{k}}\right)|i\rangle\langle j|\right]\right. \\
& \left.\times\left[\sum_{i, j, \mathbf{k}}^{i \neq j} \frac{g_{\mathbf{k}, i j} \xi_{k, i j}}{\omega_{k}}\left(b_{\mathbf{k}}^{\dagger}-b_{\mathbf{k}}\right)|i\rangle\langle j|\right]\right\}+\frac{1}{2}\left\{\left[\sum_{i, j, \mathbf{k}}^{i \neq j} \frac{g_{\mathbf{k}, i j} \xi_{k, i j}}{\omega_{k}}\left(b_{\mathbf{k}}^{\dagger}-b_{\mathbf{k}}\right)|i\rangle\langle j|\right]\left[\sum_{i, j, \mathbf{k}}^{i \neq j} \frac{g_{\mathbf{k}, i j} \xi_{k, i j}}{\omega_{k}}\left(\omega_{j}-\omega_{i}\right)\left(b_{\mathbf{k}}^{\dagger}-b_{\mathbf{k}}\right)|i\rangle\langle j|\right]\right. \\
& \left.-\left[\sum_{i \neq j, \mathbf{k}}^{i \neq j} \frac{g_{\mathbf{k}, i j} \xi_{k, i j}}{\omega_{k}}\left(\omega_{j}-\omega_{i}\right)\left(b_{\mathbf{k}}^{\dagger}-b_{\mathbf{k}}\right)|i\rangle\langle j|\right]\left[\sum_{i, j, \mathbf{k}}^{i \neq j} \frac{g_{\mathbf{k}, i j} \xi_{k, i j}}{\omega_{k}}\left(b_{\mathbf{k}}^{\dagger}-b_{\mathbf{k}}\right)|i\rangle\langle j|\right]\right\}=F_{1}+F_{2},
\end{aligned}
$$

where

$$
\begin{aligned}
F_{1}= & \frac{1}{2}\left\{-\left[\sum_{i, j, \mathbf{k}}^{i \neq j} \frac{g_{\mathbf{k}, i j} \xi_{k, i j}}{\omega_{k}}\left(b_{\mathbf{k}}^{\dagger}-b_{\mathbf{k}}\right)|i\rangle\langle j|\right]\left[\sum_{i, j, \mathbf{k}}^{i \neq j} \frac{g_{\mathbf{k}, i j} \xi_{k, i j}}{\omega_{k}}\left(b_{\mathbf{k}}^{\dagger}+b_{\mathbf{k}}\right)|i\rangle\langle j|\right]\right. \\
& \left.+\left[\sum_{i, j, \mathbf{k}}^{i \neq j} \frac{g_{\mathbf{k}, i j} \xi_{k, i j}}{\omega_{k}}\left(b_{\mathbf{k}}^{\dagger}+b_{\mathbf{k}}\right)|i\rangle\langle j|\right]\left[\sum_{i, j, \mathbf{k}}^{i \neq j} \frac{g_{\mathbf{k}, i j} \xi_{k, i j}}{\omega_{k}}\left(b_{\mathbf{k}}^{\dagger}-b_{\mathbf{k}}\right)|i\rangle\langle j|\right]\right\}
\end{aligned}
$$

and

$$
\begin{aligned}
F_{2}= & \frac{1}{2}\left\{\left[\sum_{i, j, \mathbf{k}}^{i \neq j} \frac{g_{\mathbf{k}, i j} \xi_{k, i j}}{\omega_{k}}\left(b_{\mathbf{k}}^{\dagger}-b_{\mathbf{k}}\right)|i\rangle\langle j|\right]\left[\sum_{i, j, \mathbf{k}}^{i \neq j} \frac{g_{\mathbf{k}, i j} \xi_{k, i j}}{\omega_{k}}\left(\omega_{j}-\omega_{i}\right)\left(b_{\mathbf{k}}^{\dagger}-b_{\mathbf{k}}\right)|i\rangle\langle j|\right]\right. \\
& \left.-\left[\sum_{i, j, \mathbf{k}}^{i \neq j} \frac{g_{\mathbf{k}, i j} \xi_{k, i j}}{\omega_{k}}\left(\omega_{j}-\omega_{i}\right)\left(b_{\mathbf{k}}^{\dagger}-b_{\mathbf{k}}\right)|i\rangle\langle j|\right]\left[\sum_{i, j, \mathbf{k}}^{i \neq j} \frac{g_{\mathbf{k}, i j} \xi_{k, i j}}{\omega_{k}}\left(b_{\mathbf{k}}^{\dagger}-b_{\mathbf{k}}\right)|i\rangle\langle j|\right]\right\} .
\end{aligned}
$$

For $F_{1}$, we have

$$
\begin{aligned}
F_{1} & =\frac{1}{2}\left\{-\left[\sum_{i, j, \mathbf{k}}^{i \neq j} \frac{g_{\mathbf{k}, i j} \xi_{k, i j}}{\omega_{k}}\left(b_{\mathbf{k}}^{\dagger}-b_{\mathbf{k}}\right)|i\rangle\langle j|\right]\left[\sum_{i, j, \mathbf{k}}^{i \neq j} \frac{g_{\mathbf{k}, i j} \xi_{k, i j}}{\omega_{k}}\left(b_{\mathbf{k}}^{\dagger}+b_{\mathbf{k}}\right)|i\rangle\langle j|\right]+\left[\sum_{i, j, \mathbf{k}}^{i \neq j} \frac{g_{\mathbf{k}, i j} \xi_{k, i j}}{\omega_{k}}\left(b_{\mathbf{k}}^{\dagger}+b_{\mathbf{k}}\right)|i\rangle\langle j|\right]\left[\sum_{i, j, \mathbf{k}}^{i \neq j} \frac{g_{\mathbf{k}, i j} \xi_{k, i j}}{\omega_{k}}\left(b_{\mathbf{k}}^{\dagger}-b_{\mathbf{k}}\right)|i\rangle\langle j|\right]\right\} \\
& =-\frac{1}{2} \sum_{\mathbf{k}}\left[\left(b_{\mathbf{k}}^{\dagger}-b_{\mathbf{k}}\right)\left(b_{\mathbf{k}}^{\dagger}+b_{\mathbf{k}}\right)-\left(b_{\mathbf{k}}^{\dagger}+b_{\mathbf{k}}\right)\left(b_{\mathbf{k}}^{\dagger}-b_{\mathbf{k}}\right)\right]\left(\sum_{i, j}^{i \neq j} \frac{g_{\mathbf{k}, i j} \xi_{k, i j} \mid}{\omega_{k}}|i\rangle\langle j|\right)\left(\sum_{i^{\prime}, j^{\prime}}^{i^{\prime} \neq j^{\prime}} \frac{g_{\mathbf{k}, i^{\prime} j^{\prime}} \xi_{k, i^{\prime} j^{\prime}}}{\omega_{k}}\left|i^{\prime}\right\rangle\left\langle j^{\prime}\right|\right) \\
& =\sum_{\mathbf{k}} \sum_{i, j}^{i \neq j} \frac{g_{\mathbf{k}, i j} \xi_{k, i j} \sum^{i^{\prime} \neq j^{\prime}}}{\omega_{k}} \sum_{i^{\prime}, j^{\prime}} \frac{g_{\mathbf{k}, i^{\prime} j^{\prime}} \xi_{k, i^{\prime} j^{\prime}}}{\omega_{k}}|i\rangle\left\langle j \| i^{\prime}\right\rangle\left\langle j^{\prime}\right| \\
& =\sum_{\mathbf{k}} \sum_{i \neq j, j, j^{\prime}}^{i \neq j \neq j^{\prime}} \frac{g_{\mathbf{k}, i j} \xi_{k, i j} g_{\mathbf{k}, j j^{\prime}} \xi_{k, j j^{\prime}}}{\omega_{k}^{2}}|i\rangle\left\langle j^{\prime}\left|=\sum_{i, j, \mathbf{k}}^{i \neq j} \frac{g_{\mathbf{k}, i j}^{2} \xi_{k, i j}^{2}}{\omega_{k}}\right| i\right\rangle\langle i|
\end{aligned}
$$

where Eq. (7) was used, and in the last step the off-diagonal terms were neglected. For $F_{2}$, we have

$$
\begin{aligned}
F_{2}= & \frac{1}{2}\left\{\left[\sum_{i, j, \mathbf{k}}^{i \neq j} \frac{g_{\mathbf{k}, i j} \xi_{k, i j}}{\omega_{k}}\left(b_{\mathbf{k}}^{\dagger}-b_{\mathbf{k}}\right)|i\rangle\langle j|\right]\left[\sum_{i, j, \mathbf{k}}^{i \neq j} \frac{g_{\mathbf{k}, i j} \xi_{k, i j}}{\omega_{k}}\left(\omega_{j}-\omega_{i}\right)\left(b_{\mathbf{k}}^{\dagger}-b_{\mathbf{k}}\right)|i\rangle\langle j|\right]\right. \\
& \left.-\left[\sum_{i, j, \mathbf{k}}^{i \neq j} \frac{g_{\mathbf{k}, i j} \xi_{k, i j}}{\omega_{k}}\left(\omega_{j}-\omega_{i}\right)\left(b_{\mathbf{k}}^{\dagger}-b_{\mathbf{k}}\right)|i\rangle\langle j|\right]\left[\sum_{i, j, \mathbf{k}}^{i \neq j} \frac{g_{\mathbf{k}, i j} \xi_{k, i j}}{\omega_{k}}\left(b_{\mathbf{k}}^{\dagger}-b_{\mathbf{k}}\right)|i\rangle\langle j|\right]\right\} \\
= & \frac{1}{2}\left\{\sum_{\mathbf{k}}\left(b_{\mathbf{k}}^{\dagger}-b_{\mathbf{k}}\right)^{2}\left(\sum_{i, j}^{i \neq j} \frac{g_{\mathbf{k}, i j} \xi_{k, i j}}{\omega_{k}}|i\rangle\langle j|\right)\left[\sum_{i^{\prime}, j^{\prime}}^{i^{\prime} \neq j^{\prime}} \frac{g_{\mathbf{k}, i^{\prime} j^{\prime}} \xi_{k, i^{\prime} j^{\prime}}}{\omega_{k}}\left(\omega_{j^{\prime}}-\omega_{i^{\prime}}\right)\left|i^{\prime}\right\rangle\left\langle j^{\prime}\right|\right]\right.
\end{aligned}
$$




$$
\begin{aligned}
& \left.-\left[\sum_{i, j, \mathbf{k}}^{i \neq j} \frac{g_{\mathbf{k}, i j} \xi_{k, i j}}{\omega_{k}}\left(\omega_{j}-\omega_{i}\right)|i\rangle\langle j|\right]\left(\sum_{i, j, \mathbf{k}}^{i \neq j} \frac{g_{\mathbf{k}, i j} \xi_{k, i j}}{\omega_{k}}|i\rangle\langle j|\right)\right\} \\
= & \frac{1}{2} \sum_{\mathbf{k}}\left(b_{\mathbf{k}}^{\dagger}-b_{\mathbf{k}}\right)^{2}\left[\sum_{i, j, j^{\prime}}^{i \neq j, j \neq j^{\prime}} \frac{g_{\mathbf{k}, i j} \xi_{k, i j}}{\omega_{k}^{2}} g_{\mathbf{k}, j j^{\prime}} \xi_{k, j j^{\prime}}\left(\omega_{j^{\prime}}-\omega_{j}\right)|i\rangle\left\langle j^{\prime}\left|-\sum_{i, j, j^{\prime}}^{i \neq j, j \neq j^{\prime}} \frac{g_{\mathbf{k}, i j} \xi_{k, i j}}{\omega_{k}^{2}} g_{\mathbf{k}, j j^{\prime}} \xi_{k, j j^{\prime}}\left(\omega_{j}-\omega_{j^{\prime}}\right)\right| i\right\rangle\left\langle j^{\prime}\right|\right] .
\end{aligned}
$$

Neglecting the off-diagonal terms in the atomic operators, we have

$$
\begin{aligned}
F_{2} & =\frac{1}{2} \sum_{\mathbf{k}}\left(b_{\mathbf{k}}^{\dagger}-b_{\mathbf{k}}\right)^{2}\left[\sum_{i, j}^{i \neq j} \frac{g_{\mathbf{k}, i j}^{2} \xi_{k, i j}^{2}}{\omega_{k}^{2}}\left(\omega_{i}-\omega_{j}\right)|i\rangle\left\langle i\left|-\sum_{i, j}^{i \neq j} \frac{g_{\mathbf{k}, i j}^{2} \xi_{k, i j}^{2}}{\omega_{k}^{2}}\left(\omega_{j}-\omega_{i}\right)\right| i\right\rangle\langle i|\right] \\
& =\frac{1}{2} \sum_{\mathbf{k}}\left(b_{\mathbf{k}}^{\dagger}-b_{\mathbf{k}}\right)^{2}\left[\sum_{i, j}^{i \neq j} \frac{g_{\mathbf{k}, i j}^{2} \xi_{k, i j}^{2}}{\omega_{k}^{2}}\left(\omega_{i}-\omega_{j}\right)|i\rangle\left\langle i\left|+\sum_{i, j}^{i \neq j} \frac{g_{\mathbf{k}, i j}^{2} \xi_{k, i j}^{2}}{\omega_{k}^{2}}\left(\omega_{i}-\omega_{j}\right)\right| i\right\rangle\langle i|\right] \\
& =\sum_{\mathbf{k}}\left(b_{\mathbf{k}}^{\dagger}-b_{\mathbf{k}}\right)^{2} \sum_{i, j}^{i \neq j} \frac{g_{\mathbf{k}, i j}^{2} \xi_{k, i j}^{2}}{\omega_{k}^{2}}\left(\omega_{i}-\omega_{j}\right)|i\rangle\langle i| .
\end{aligned}
$$

If we neglect the off-diagonal terms $b_{\mathbf{k}}^{\dagger} b_{\mathbf{k}}^{\dagger}, b_{\mathbf{k}} b_{\mathbf{k}}$ and also use $b_{\mathbf{k}}^{\dagger} b_{\mathbf{k}}\left|\{0\}_{\mathbf{k}}\right\rangle=0$ in our consideration for the renormalization by the vacuum, $F_{2}$ can be written as

Combining $F_{1}$ and $F_{2}$, we have

$$
F_{2}=\sum_{i, j, \mathbf{k}}^{i \neq j} \frac{g_{\mathbf{k} . i j}^{2} \xi_{k, i j}^{2}}{\omega_{k}^{2}}\left(\omega_{j}-\omega_{i}\right)|i\rangle\langle i| .
$$

$$
\frac{1}{2}\left[i S,\left[i S, H_{0}\right]\right]=\sum_{i, j, \mathbf{k}}^{i \neq j} \frac{g_{\mathbf{k} . i j}^{2} \xi_{k, i j}^{2}}{\omega_{k}}|i\rangle\left\langle i\left|+\sum_{i, j, \mathbf{k}}^{i \neq j} \frac{g_{\mathbf{k} . i j}^{2} \xi_{k, i j}^{2}}{\omega_{k}^{2}}\left(\omega_{j}-\omega_{i}\right)\right| i\right\rangle\langle i| .
$$

From Eqs. (A3) and (A9), we have

$$
\begin{aligned}
H_{2}^{S} & =\left[i S, H_{1}\right]+\frac{1}{2}\left[i S,\left[i S, H_{0}\right]\right] \\
& =-2 \sum_{i, j, \mathbf{k}}^{i \neq j} \frac{g_{\mathbf{k}, i j}^{2} \xi_{k, i j}}{\omega_{k}}|i\rangle\left\langle i\left|+\sum_{i, j, \mathbf{k}}^{i \neq j} \frac{g_{\mathbf{k} . i j}^{2} \xi_{k, i j}^{2}}{\omega_{k}}\right| i\right\rangle\left\langle i\left|+\sum_{i, j, \mathbf{k}}^{i \neq j} \frac{g_{\mathbf{k} . i j}^{2} \xi_{k, i j}^{2}}{\omega_{k}^{2}}\left(\omega_{j}-\omega_{i}\right)\right| i\right\rangle\langle i| \\
& =-\sum_{i, j, \mathbf{k}}^{i \neq j} \frac{g_{\mathbf{k}, i j}^{2}}{\omega_{k}}\left[2 \xi_{k, i j}-\xi_{k, i j}^{2}-\frac{\xi_{k, i j}^{2}}{\omega_{k}}\left(\omega_{j}-\omega_{i}\right)\right]|i\rangle\langle i|,
\end{aligned}
$$

which is Eq. (10) in the text.

\section{APPENDIX C: THE NONDYNAMIC SHIFT}

By using Eqs. (B10) and (11), we have

$$
\begin{aligned}
& \Delta E_{n d}^{(i)}=\left\langle i\left|H_{2}^{S}-E_{s e}\right| i\right\rangle=\omega_{i}-\omega_{i}^{\prime} \\
& =-\sum_{j} \sum_{\mathbf{k}} \frac{g_{\mathbf{k}, i j}^{2}}{\omega_{k}}\left\{2 \xi_{k, i j}-\xi_{k, i j}^{2}-1-\xi_{k, i j}^{2} \frac{\omega_{j i}}{\omega_{k}}\right\} \\
& =\sum_{j} \sum_{\mathbf{k}} \frac{g_{\mathbf{k}, i j}^{2}}{\omega_{k}}\left\{\frac{\omega_{i j}^{2}}{\left(\omega_{k}+\left|\omega_{i j}\right|\right)^{2}}+\frac{\omega_{k} \omega_{j i}}{\left(\omega_{k}+\left|\omega_{i j}\right|\right)^{2}}\right\} \\
& =\sum_{j} \sum_{\mathbf{k}} \frac{g_{\mathbf{k}, i j}^{2}}{\omega_{k}} \frac{\omega_{i j}^{2}}{\left(\omega_{k}+\left|\omega_{i j}\right|\right)^{2}}+\sum_{\mathbf{k}} \sum_{j>i} \frac{g_{\mathbf{k}, i j}^{2}}{\omega_{k}} \frac{\omega_{k} \omega_{j i}+\omega_{j i}^{2}-\omega_{j i}^{2}}{\left(\omega_{k}+\left|\omega_{i j}\right|\right)^{2}}-\sum_{\mathbf{k}} \sum_{j<i} \frac{g_{\mathbf{k}, i j}^{2}}{\omega_{k}} \frac{\omega_{k} \omega_{i j}+\omega_{i j}^{2}-\omega_{i j}^{2}}{\left(\omega_{k}+\left|\omega_{i j}\right|\right)^{2}} \\
& =\sum_{j} \sum_{\mathbf{k}} \frac{g_{\mathbf{k}, i j}^{2}}{\omega_{k}} \frac{\omega_{i j}^{2}}{\left(\omega_{k}+\left|\omega_{i j}\right|\right)^{2}}+\sum_{\mathbf{k}} \sum_{j>i} \frac{g_{\mathbf{k}, i j}^{2}}{\omega_{k}} \frac{(-1) \omega_{j i}^{2}}{\left(\omega_{k}+\left|\omega_{i j}\right|\right)^{2}}+\sum_{\mathbf{k}} \sum_{j<i} \frac{g_{\mathbf{k}, i j}^{2}}{\omega_{k}} \frac{\omega_{i j}^{2}}{\left(\omega_{k}+\left|\omega_{i j}\right|\right)^{2}}+\sum_{\mathbf{k}} \sum_{j>i} \frac{g_{\mathbf{k}, i j}^{2}}{\omega_{k}} \frac{\omega_{k} \omega_{j i}+\omega_{j i}^{2}}{\left(\omega_{k}+\left|\omega_{i j}\right|\right)^{2}} \\
& -\sum_{k} \sum_{j<i} \frac{g_{\mathbf{k}, i j}^{2}}{\omega_{k}} \frac{\omega_{k} \omega_{i j}+\omega_{i j}^{2}}{\left(\omega_{k}+\left|\omega_{i j}\right|\right)^{2}}
\end{aligned}
$$




$$
\begin{aligned}
& =\sum_{\mathbf{k}} \sum_{j<i} \frac{2 g_{\mathbf{k}, i j}^{2}}{\omega_{k}} \frac{\omega_{i j}^{2}}{\left(\omega_{k}+\mid \omega_{i j}\right)^{2}}+\sum_{\mathbf{k}} \sum_{j} \frac{g_{\mathbf{k}, i j}^{2}}{\omega_{k}} \frac{\omega_{j i}}{\left(\omega_{k}+\left|\omega_{i j}\right|\right)} \\
& =\sum_{\mathbf{k}} \sum_{j<i} \frac{g_{\mathbf{k}, i j}^{2}}{\omega_{k}} \frac{\left(\omega_{i j}^{2}-\omega_{i j} \omega_{k}\right)}{\left(\omega_{k}+\omega_{i j}\right)^{2}}+\sum_{\mathbf{k}} \sum_{j>i} \frac{g_{\mathbf{k}, i j}^{2}}{\omega_{k}} \frac{\omega_{j i}}{\left(\omega_{k}+\omega_{j i}\right)}=\sum_{k} \sum_{j \neq i} \frac{g_{k, i j}^{2}}{\omega_{k}} \frac{\omega_{j i}\left(\omega_{j i}+\omega_{k}\right)}{\left(\omega_{k}+\mid \omega_{i j}\right)^{2}},
\end{aligned}
$$

which is Eq. (14b) in the text. For the long-time limit, the dependent dynamic shift $\Delta E_{d y n}(\infty)$ can be easily found from Eqs. (28) and (8a) in the text,

$$
\Delta E_{d y n}^{(i)}(\infty)=\sum_{\mathbf{k}} \sum_{j<i} \frac{4 \omega_{i j}^{2} g_{\mathbf{k}, i j}^{2} \xi_{k, i j}^{2}}{\omega_{k}^{2}\left(\omega_{i j}-\omega_{k}\right)}=\sum_{\mathbf{k}} \sum_{j<i} \frac{4 \omega_{i j}^{2} g_{\mathbf{k}, i j}^{2}}{\left(\omega_{i j}-\omega_{k}\right)\left(\omega_{k}+\left|\omega_{i j}\right|\right)^{2}} .
$$

Combining Eqs. (B1) and (B2), we have the total energy shift $\Delta E_{\text {total }}^{(i)}(\infty)$ for the long-time limit,

$$
\begin{aligned}
& \Delta E_{\text {total }}^{(i)}=\Delta E_{n d}^{(i)}+\Delta E_{d y n}^{(i)} \\
& =\sum_{\mathbf{k}} \sum_{j<i} \frac{2 g_{\mathbf{k}, i j}^{2}}{\omega_{k}} \frac{\omega_{i j}^{2}}{\left(\omega_{k}+\mid \omega_{i j}\right)^{2}}+\sum_{\mathbf{k}} \sum_{j} \frac{g_{\mathbf{k}, i j}^{2}}{\omega_{k}} \frac{\omega_{j i}}{\left(\omega_{k}+\left|\omega_{i j}\right|\right)}+\sum_{\mathbf{k}} \sum_{j<i} \frac{4 \omega_{i j}^{2} g_{\mathbf{k}, i j}^{2}}{\left(\omega_{i j}-\omega_{k}\right)\left(\omega_{k}+\left|\omega_{i j}\right|\right)^{2}} \\
& =\sum_{\mathbf{k}} \sum_{j} \frac{g_{\mathbf{k}, i j}^{2}}{\omega_{k}} \frac{\omega_{j i}}{\left(\omega_{k}+\left|\omega_{i j}\right|\right)}+\sum_{\mathbf{k}} \sum_{j<i} \frac{2 g_{\mathbf{k}, i j}^{2}}{\omega_{k}} \frac{\omega_{i j}^{2}}{\left(\omega_{k}+\left|\omega_{i j}\right|\right)^{2}}-\sum_{\mathbf{k}} \sum_{j<i} \frac{4 \omega_{i j}^{2} g_{\mathbf{k}, i j}^{2}}{\left(\omega_{k}-\omega_{i j}\right)\left(\omega_{k}+\left|\omega_{i j}\right|\right)^{2}} \\
& =\sum_{\mathbf{k}} \sum_{j} \frac{g_{\mathbf{k}, i j}^{2}}{\omega_{k}} \frac{\omega_{j i}}{\left(\omega_{k}+\left|\omega_{i j}\right|\right)}+\sum_{\mathbf{k}} \sum_{j<i} \frac{g_{\mathbf{k}, i j}^{2}}{\omega_{k}} \frac{\left(\omega_{i j}\right)^{2}}{\left(\omega_{k}+\left|\omega_{i j}\right|\right)^{2}}\left(2-\frac{4 \omega_{k}}{\omega_{k}-\omega_{i j}}\right) \\
& =\sum_{\mathbf{k}} \sum_{j} \frac{g_{\mathbf{k}, i j}^{2}}{\omega_{k}} \frac{\omega_{j i}}{\left(\omega_{k}+\left|\omega_{i j}\right|\right)}+\sum_{\mathbf{k}} \sum_{j<i} \frac{g_{\mathbf{k}, i j}^{2}}{\omega_{k}} \frac{2 \omega_{i j}^{2}}{\left(\omega_{k}+\mid \omega_{i j}\right)^{2}}\left[-\frac{\omega_{k}+\omega_{i j}}{\omega_{k}-\omega_{i j}}\right] \\
& =\sum_{\mathbf{k}} \sum_{j} \frac{g_{\mathbf{k}, i j}^{2}}{\omega_{k}} \frac{\omega_{j i}}{\left(\omega_{k}+\left|\omega_{i j}\right|\right)}+\sum_{\mathbf{k}} \sum_{j<i} \frac{g_{\mathbf{k}, i j}^{2}}{\omega_{k}} \frac{2\left(\omega_{i j}\right)^{2}}{\left(\omega_{k}+\left|\omega_{i j}\right|\right)\left(\omega_{i j}-\omega_{k}\right)} \\
& =\sum_{\mathbf{k}} \sum_{j>i} \frac{g_{\mathbf{k}, i j}^{2}}{\omega_{k}} \frac{\omega_{j i}}{\left(\omega_{k}+\left|\omega_{i j}\right|\right)}+\sum_{\mathbf{k}} \sum_{j<i} \frac{g_{\mathbf{k}, i j}^{2}}{\omega_{k}} \frac{\omega_{j i}}{\left(\omega_{k}+\left|\omega_{i j}\right|\right)}+\sum_{\mathbf{k}} \sum_{j<i} \frac{g_{\mathbf{k}, i j}^{2}}{\omega_{k}} \frac{2 \omega_{i j}^{2}}{\left(\omega_{k}+\left|\omega_{i j}\right|\right)\left(\omega_{i j}-\omega_{k}\right)} \\
& =\sum_{\mathbf{k}} \sum_{j>i} \frac{g_{\mathbf{k}, i j}^{2}}{\omega_{k}} \frac{\omega_{j i}}{\left(\omega_{k}+\omega_{j i}\right)}+\sum_{\mathbf{k}} \sum_{j<i} \frac{g_{\mathbf{k}, i j}^{2}}{\omega_{k}} \frac{\omega_{i j}}{\left(\omega_{i j}-\omega_{k}\right)} \\
& =\sum_{\mathbf{k}} \sum_{j>i} \frac{g_{\mathbf{k}, i j}^{2}}{\omega_{k}} \frac{\omega_{j i}}{\left(\omega_{k}+\omega_{j i}\right)}+\sum_{\mathbf{k}} \sum_{j<i} \frac{g_{\mathbf{k}, i j}^{2}}{\omega_{k}} \frac{\omega_{j i}}{\left(\omega_{j i}+\omega_{k}\right)}=\sum_{\mathbf{k}} \sum_{j \neq i} \frac{g_{\mathbf{k}, i j}^{2}}{\omega_{k}} \frac{\omega_{i j}}{\left(\omega_{i j}-\omega_{k}\right)},
\end{aligned}
$$

which is Eq. (30) in the text.

[1] H. Zheng, S. Y. Zhu, and M. S. Zubairy, Phys. Rev. Lett. 101, 200404 (2008).

[2] W. H. Louisell, Quantum Statistical Properties of Radiation (John Wiley, New York, 1973), Chap. 5.

[3] C. Cohen-Tannoudji, J. Dupont-Poc, and G. Grynberg, AtomPhoton Interactions: Basic Processes and Applications (Wiley, New York, 1992).

[4] A. G. Kofman and G. Kurizki, Phys. Rev. Lett. 93, 130406 (2004).

[5] B. Misra and E. C. G. Sudarshan, J. Math. Phys. 18, 756 (1977); V. Frerichs and A. Schenzle, Phys. Rev. A 44, 1962 (1991); L. S. Schulman, ibid. 57, 1509 (1998).

[6] W. M. Itano, D. J. Heinzen, J. J. Bollinger, and D. J. Wineland, Phys. Rev. A 41, 2295 (1990); M. C. Fischer, B. GutierrezMedina, and M. G. Raizen, Phys. Rev. Lett. 87, 040402 (2001).
[7] S. Maniscalco, J. Piilo, and K.-A. Suominen, Phys. Rev. Lett. 97, 130402 (2006).

[8] A. G. Kofman and G. Kurizki, Nature (London) 405, 546 (2000); Phys. Rev. A 54, R3750 (1996).

[9] P. Facchi and S. Pascazio, Phys. Lett. A 241, 139 (1998).

[10] P. Facchi and S. Pascazio, Phys. Rev. A 62, 023804 (2000).

[11] P. Facchi, H. Nakazato, and S. Pascazio, Phys. Rev. Lett. 86, 2699 (2001).

[12] N. Erez, G. Gordon, M. Nest, and G. Kurizki, Nature (London) 452, 724 (2008).

[13] R. Loudon and S. M. Barnett, J. Phys. B 39, S555 (2006).

[14] H. A. Bethe, Phys. Rev. 72, 339 (1947).

[15] M. O. Scully and M. S. Zubairy, Quantum Optics (University Press, Cambridge, 1997).

[16] M. Lieber, Phys. Rev. 174, 2037 (1968). 